\title{
Impact of elastic ankle exoskeleton stiffness on neuromechanics and energetics of human walking across multiple speeds
}

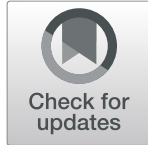

Richard W. Nuckols ${\text {, } 2,3^{*}}^{\text {(D) }}$ and Gregory S. Sawicki ${ }^{1,4^{*}}$

\begin{abstract}
Background: Elastic ankle exoskeletons with intermediate stiffness springs in parallel with the human plantarflexors can reduce the metabolic cost of walking by $\sim 7 \%$ at $1.25 \mathrm{~m} \mathrm{~s}^{-1}$. In a move toward 'real-world' application, we examined whether the unpowered approach has metabolic benefit across a range of walking speeds, and if so, whether the optimal exoskeleton stiffness was speed dependent. We hypothesized that, for any walking speed, there would be an optimal ankle exoskeleton stiffness - not too compliant and not too stiff - that minimizes the user's metabolic cost. In addition, we expected the optimal stiffness to increase with walking speed.
\end{abstract}

Methods: Eleven participants walked on a level treadmill at 1.25, 1.50, and $1.75 \mathrm{~m} \mathrm{~s}^{-1}$ while we used a state-of-theart exoskeleton emulator to apply bilateral ankle exoskeleton assistance at five controlled rotational stiffnesses $\left(k_{\text {exo }}=0,50,100,150,250 \mathrm{Nm} \mathrm{rad}^{-1}\right)$. We measured metabolic cost, lower-limb joint mechanics, and EMG of muscles crossing the ankle, knee, and hip.

Results: Metabolic cost was significantly reduced at the lowest exoskeleton stiffness $\left(50 \mathrm{Nm} \mathrm{rad}^{-1}\right)$ for assisted walking at both $1.25(4.2 \% ; p=0.0162)$ and $1.75 \mathrm{~m} \mathrm{~s}^{-1}(4.7 \% ; p=0.0045)$. At these speeds, the metabolically optimal exoskeleton stiffness provided peak assistive torques of $\sim 0.20 \mathrm{Nm} \mathrm{kg}^{-1}$ that resulted in reduced biological ankle moment of $\sim 12 \%$ and reduced soleus muscle activity of $\sim 10 \%$. We found no stiffness that could reduce the metabolic cost of walking at $1.5 \mathrm{~m} \mathrm{~s}^{-1}$. Across all speeds, the non-weighted sum of soleus and tibialis anterior activation rate explained the change in metabolic rate due to exoskeleton assistance $\left(p<0.05 ; R^{2}>0.56\right)$.

Conclusions: Elastic ankle exoskeletons with low rotational stiffness reduce users' metabolic cost of walking at slow and fast but not intermediate walking speed. The relationship between the non-weighted sum of soleus and tibialis activation rate and metabolic cost $\left(R^{2}>0.56\right)$ indicates that muscle activation may drive metabolic demand. Future work using simulations and ultrasound imaging will get 'under the skin' and examine the interaction between exoskeleton stiffness and plantarflexor muscle dynamics to better inform stiffness selection in human-machine systems.

Keywords: Locomotion, Wearable robotics, Plantarflexors, Spring-loaded, Lower-limb joints, Biomechanics, Metabolic cost, Compliant

\footnotetext{
*Correspondence: rnuckols@seas.harvard.edu;

gregory.sawicki@me.gatech.edu

'Joint Department of Biomedical Engineering, UNC Chapel Hill and NC State

University, Raleigh, NC, USA

Full list of author information is available at the end of the article
}

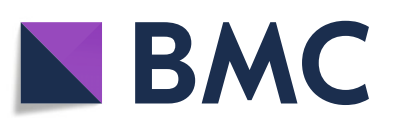

(- The Author(s). 2020 Open Access This article is licensed under a Creative Commons Attribution 4.0 International License, which permits use, sharing, adaptation, distribution and reproduction in any medium or format, as long as you give appropriate credit to the original author(s) and the source, provide a link to the Creative Commons licence, and indicate if changes were made. The images or other third party material in this article are included in the article's Creative Commons licence, unless indicated otherwise in a credit line to the material. If material is not included in the article's Creative Commons licence and your intended use is not permitted by statutory regulation or exceeds the permitted use, you will need to obtain permission directly from the copyright holder. To view a copy of this licence, visit http://creativecommons.org/licenses/by/4.0/. The Creative Commons Public Domain Dedication waiver (http://creativecommons.org/publicdomain/zero/1.0/) applies to the data made available in this article, unless otherwise stated in a credit line to the data. 


\section{Background}

The field of wearable robotic devices for improving locomotion performance is quickly expanding. In fact, it has been just over 7 years since a tethered, pneumatically powered ankle exoskeleton was shown to reduce the metabolic cost of walking by $6 \%$ below normal, demonstrating for the first time that a wearable robotic device could improve the economy of human locomotion [1]. Since that milestone study that 'broke the metabolic cost barrier', the metabolic benefit of exoskeleton assistance has been steadily ticking upward [2]. For example, the benefit of powered ankle assistance was successfully transferred to a portable device that can reduce the metabolic cost of walking by up to $11 \%$ compared to normal [3-5]. Ongoing experiments using tethered ankle exoskeletons to tune the timing and magnitude of torque assistance have now shown metabolic benefit up to $12 \%$ compared to normal walking [6] and as much as $24 \%$ for a unilateral system when compared to zerotorque mode [7]. These experiments suggest that savings of $>=30 \%$ for bilateral ankle assistance with optimized torque profiles may be possible for a portable system if the cost of carrying the device and its actuators can be minimized. These ankle-based and powered lower-limb exoskeleton systems targeting other joints $[8,9]$ accomplish the goal of reducing metabolic cost by transferring net mechanical energy to the user. Alternatively, our research has shown that it is possible to use an unpowered, passive-elastic ankle exoskeleton to reduce the metabolic cost of walking by $7.2 \%$ while delivering no net mechanical work [10].

Regardless of differences in the method that lowerlimb exoskeleton systems use to deliver mechanical assistance (e.g., passive vs. active; myoelectric vs. impedance control), a common thread across studies has been the limited range of gait conditions examined when evaluating their performance. Most exoskeleton devices have been tested on level ground at one single speed. Although the level-ground, single speed combination has proven an important benchmark used in cross-study comparisons, if the goal is to employ these devices in 'real-world' scenarios, then further work in evaluation of exoskeleton assistance under a more comprehensive set of gait conditions is needed. Our world is dynamic - we walk, run, change speeds, and move up and downhill. Thus, it is surprising that only a few exoskeleton devices have been extensively tested under 'non-baseline' conditions such as load carriage [4], incline walking [11, 12], and intermediate speed walking $[4,13]$. In fact, to our knowledge, no study has explicitly evaluated the effect of walking speed on human-exoskeleton neuromechanics and energetics within a single study. Here, we use a state-of-the-art, tethered, powered exoskeleton system to emulate the dynamics of an unpowered elastic ankle exoskeleton based on our previous work [10], confirm its performance at a single speed, and then extend our evaluation to examine performance across a functionally relevant range of walking speeds. Establishing the limits of passive systems in a rigorous and focused scientific manner is important in and of itself, but will also inform the development of devices that incorporate both passive and active components (i.e., semi-active), which have gained popularity in recent years due to their compact and lightweight form-factor, low power requirements and favorable dynamic response [14-17].

The specific goal of this work was to evaluate how the coupling between walking speed and ankle exoskeleton rotational stiffness influences the user's neuromechanics and energetics. Our previous research indicates that bilateral elastic ankle exoskeletons that place intermediate stiffness springs in parallel with the human triceps surae and Achilles' tendon can reduce metabolic cost during level walking at $1.25 \mathrm{~m} \mathrm{~s}^{-1}$ by up to $7 \%$ [10]. Whether this same parallel stiffness is optimal at other walking speeds has yet to be determined. As a starting point for developing hypotheses for how the metabolically optimal ankle exoskeleton stiffness ought to change with walking speed, we refer to the biological system for inspiration.

The ankle is an important contributor to mechanical power output in both walking and running over a range of gait speeds. In normal gait, the muscle tendon unit (MTU) of the ankle joint efficiently contributes approximately half of the positive mechanical power generated by the legs for walking [18]. In late stance, the ankle provides almost the entirety of positive power for redirecting the center of mass [19]. In providing an efficient and powerful ankle plantarflexion moment, the muscle and tendon work in concert as series elements. In early stance, as the MTU lengthens, the Achilles tendon stretches and stores elastic energy against the clutch-like isometric triceps surae muscles [20,21]. This is followed by a rapid period of muscle contraction and tendon recoil where the tendon provides nearly half the positive power [22]. As such, the ankle joint exhibits mechanics that are similar to the loading and unloading of a torsional spring, especially at slow to medium walking speeds.

The spring-like behavior of the ankle joint can be characterized using the 'quasi-stiffness'. That is, the combined effect of the stiffness derived from tendons, active and passive elements of muscle, and other connective tissues acting across a joint can be estimated by computing the local slope of the moment-angle curve at any phase of a gait cycle. The literature detailing how biological joint quasi-stiffness changes with gait speed is not entirely clear. In a forward-dynamic walking model, the stiffness of an ankle-foot orthosis (meant to simulate a passive ankle) that minimized metabolic cost of 
transport was nearly constant over gait speeds of 0.5$0.8 \mathrm{~m} \mathrm{~s}^{-1}$. However, in this study the simulated walking speeds were much slower than preferred walking speed in able-bodied adults [23]. Experiments on humans with gait speeds of $0.75-2.63 \mathrm{~m} \mathrm{~s}^{-1}$ suggest that the biological ankle quasi-stiffness increases with increasing walking speed. However, the quasi-stiffness and relative increase in ankle quasi-stiffness with speed depends on the phase of stance in which it is evaluated (i.e., early, mid, late stance) [24]. For example, quasi-stiffness in early stance increases from approximately 280 to $500 \mathrm{Nm} \mathrm{rad}^{-1}$ with increased walking speed from 0.75 to $1.9 \mathrm{~m} \mathrm{~s}^{-1}$, while quasi-stiffness in mid and late stance increases by 250 $\mathrm{Nm} \mathrm{rad}^{-1}$ and $40 \mathrm{Nm} \mathrm{rad}^{-1}$ respectively in the same range [24].

Previously, using a portable, unpowered elastic ankle exoskeleton at a single speed $\left(1.25 \mathrm{~m} \mathrm{~s}^{-1}\right)$ we discovered a 'sweet spot' in the relationship between exoskeleton rotational stiffness and the user's metabolic cost where metabolic rate was decreased by $7.2 \%$ with an optimal stiffness of $180 \mathrm{Nm} \mathrm{rad}^{-1}$ [10]. Here, we hypothesized that, for any given walking speed, there would be a quadratic, 'bowl-shape' relationship between the user's metabolic rate and exoskeleton stiffness. In other words, at each speed, there exists an optimal exoskeleton rotational stiffness that is not too compliant and not too stiff which minimizes the user's metabolic rate. Further, in accordance with aforementioned reports indicating that biological ankle quasi-stiffness increases with speed [24], we expected that the metabolically optimal ankle exoskeleton stiffness will also increase with walking speed.

\section{Methods}

\section{Study participants}

Eleven healthy adults ( 4 female, 7 male; age: $27.7 \pm 3.3$ years; height: $1.75 \pm 0.07 \mathrm{~m}$; mass: $76.8 \pm 8.2$ $\mathrm{kg}($ mean $\pm \mathrm{SD})$ ) participated in the study. All participants signed an informed consent to participate in the study which was approved by the Institutional Review Board at University of North Carolina at Chapel Hill.

\section{Exoskeleton emulator}

The exoskeleton emulator was a custom research tool capable of providing bilateral mechanical plantarflexion assistance to the user. A similar device has been demonstrated in previous work $[25,26]$. We developed the device in our lab to efficiently and systematically test exoskeleton assistance strategies, and, in this study, we used the system to apply rotational stiffness in parallel to the human plantarflexors via bilateral ankle exoskeletons. The device consisted of three primary components: (1) bilateral ankle exoskeleton end-effectors, (2) benchtop motors and transmission, and (3) control system (Fig. 1).

Each exoskeleton end effector was a lightweight carbon fiber ankle foot orthosis (AFO) that applied plantarflexor torque to the ankle. To allow for flexible testing across participants, we designed the end-effector to be modular such that we could accommodate anthropometric variation in participants' foot, ankle and shank with four effectors (Short/Small, Short/Large, Tall/Small, Tall/Large). To align the device to the user's ankle joint center we designed a lockable slider joint to allow for anterior/ posterior positioning of the exoskeleton joint

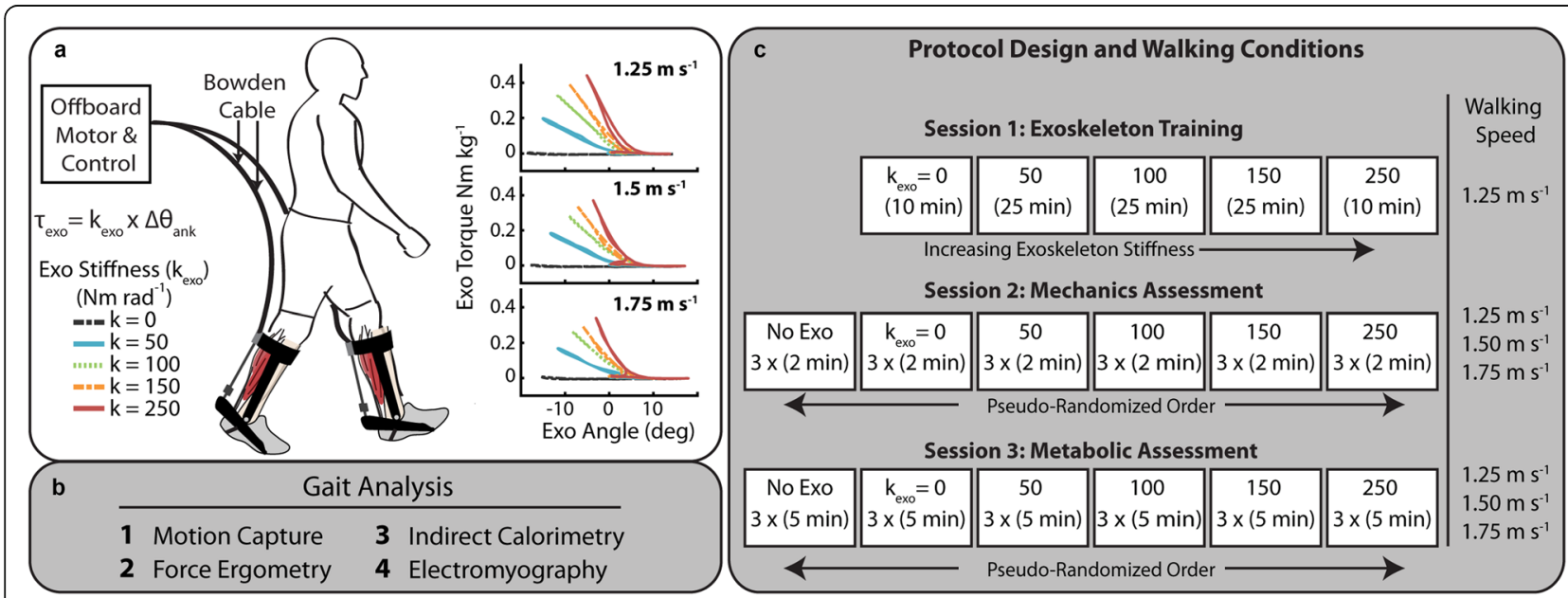

Fig. 1 Exoskeleton emulator and study protocol. (a) Representative setup of the ankle exoskeleton testing platform for evaluating rotational stiffness plantarflexion assistance. The exoskeleton provided plantarflexion torque to the bilateral ankle end-effectors through off-board motors. The controller emulated elastic rotational stiffness by imposing a torque-angle relationship control law $\left(T_{\text {exo }}=k_{\text {exo }} * \Delta \theta_{\text {ankle }}\right)$ where ankle angle was measured with a goniometer. Torque tracking was accomplished by feedback of the applied torque. The ankle exoskeleton work loops for three walking speeds show the change in slope of the work loop representing rotational stiffness (b) Biomechanical measurements performed. (c) Protocol for the three testing sessions 
center on the end-effector. The mass of the end-effector without attachment hardware was $415 \mathrm{~g}$.

Two off-board $1.61 \mathrm{~kW}$ benchtop motors (Baldor Electric Co, Fort Smith, AR) delivered plantarflexion torque to the bilateral ankle exoskeletons by applying a pulling force to the end of the exoskeleton moment arm through a flexible Bowden-cable transmission. At the end-effector, we terminated the internal cable to an inline tensile load cell (DCE-2500 N, LCM Systems, Newport, UK) and attached to the end effector moment arm $(\sim 10 \mathrm{~cm})$ through a series elastic element. The motors were positioned behind the participant at approximate shoulder height (1.37 $\mathrm{m}$ with respect to treadmill). Through pilot testing evaluation, this height and position provided the best combination of ankle joint range of motion and exoskeleton controllability and limited the interference with camera line of sight.

The exoskeleton controller consisted of a high-level module which calculated a desired torque and low-level module providing torque tracking. The system was implemented through a real-time control package (DS1103 dSpace Inc., Germany) where analog sensor data was sampled at $5 \mathrm{kHz}$ and motor command signals were generated at $500 \mathrm{~Hz}$. A high-level impedance controller determined the desired exoskeleton torque. The impedance controller was designed to emulate a physical passive elastic element capable of providing plantarflexion torque (rotational stiffness) to the ankle akin to our previous portable unpowered elastic ankle exoskeleton [10]. As such, we set the damping coefficient equal to zero, eliminating any dependence of exoskeleton torque on ankle joint angular velocity. We calculated desired torque based off a predefined rotational stiffness and the real-time ankle joint angle:

$$
\tau_{\text {exo }}=k_{\text {exo }} \times\left(\theta_{0}-\theta_{\text {ankle }}\right)
$$

where $k_{\text {exo }}$ was rotational stiffness of the exoskeleton $\left(\mathrm{Nm} \mathrm{rad}^{-1}\right), \theta_{0}$ was the onset angle ( $\left.\mathrm{rad}\right)$, and $\theta_{\text {ankle }}$ was the real-time ankle joint angle (rad). Similar to the clutch mechanism from [10], the system applied a torque only when the angle was greater than the engagement point $\left(\theta_{0}\right)$. A goniometer $(5 \mathrm{kHz}, 250 \mathrm{~Hz}$ Biometrics, Newport, UK) between the foot and shank segment of the exoskeleton measured real-time sagittal plane ankle joint angle, and the onset angle $\left(\theta_{0}\right)$ was set to the value recorded at contact of the foot with the treadmill surface. We determined gait state and heel strike by sampling vertical ground reaction force from the instrumented treadmill (Bertec, Columbus, $\mathrm{OH}$ ). At the detection of toe-off, the cable was pushed out to prevent plantarflexion torque during swing and had a similar effect to the physical pins which disengaged the clutch in the mobile device [10].
The low-level controller was a modified proportionalderivative (PD) controller where torque error was driven to zero using proportional torque error and motor velocity damping feedback in combination with inter-stride learning of torque error [27]. An inline load cell measured exoskeleton force $(250 \mathrm{~Hz}$ LP Filter) which was converted to a torque via scaling by the exoskeleton moment arm and an optical encoder (E5 Optical Encoder, US Digital, Vancouver, WA) measured motor pulley velocity. An analog velocity command was calculated and sent to the motor controller operating in velocity mode.

\section{Walking trials}

Participants completed testing over three sessions where they walked at three speeds $\left(1.25 \mathrm{~m} \mathrm{~s}^{-1}, 1.50 \mathrm{~m} \mathrm{~s}^{-1}, 1.75\right.$ $\mathrm{m} \mathrm{s}^{-1}$ ) at five ankle exoskeleton rotational stiffness conditions $\left(k_{\text {exo }}=0,50,100,150,250 \mathrm{Nm} \mathrm{rad}^{-1}\right)$. The low and mid walking speeds were chosen based off commonly used literature values for treadmill walking studies and the knowledge that preferred walking speed of humans usually falls between 1.25 and $1.5 \mathrm{~ms}^{-1}$ [28]. The fast walking speed of $1.75 \mathrm{~m} \mathrm{~s}^{-1}$ is above preferred walking speed, below the walk to run transition [29], and is a consistent increase from the previous speeds. The order of the three testing sessions was (1) exoskeleton training, (2) gait mechanics, (3) steady-state metabolic energy consumption (Fig. 1c). The imposed waiting period between each testing session was 2-7 days to allow for learning and retention [30].

\section{Training}

Previous work has demonstrated the importance of training on the acceptance of mechanical assistance in exoskeletons [31-33]. Each participant walked in the exoskeleton at $1.25 \mathrm{~m} \mathrm{~s}^{-1}$ for a total of $95 \mathrm{~min}$ over 5 training trials. The participants walked at each of intermediate stiffness conditions $\left(k_{\text {exo }}=50,100,150 \mathrm{Nm}\right.$ $\operatorname{rad}^{-1}$ ) for 25- min trials and at the lowest and highest stiffness conditions $\left(k_{\text {exo }}=0,250 \mathrm{Nm} \mathrm{rad}^{-1}\right)$ for $10 \mathrm{~min}$. We collected indirect calorimetry data for each of the trials to monitor changes in metabolic energy use over the course of the training session.

\section{Gait mechanics}

Participants walked for $2 \mathrm{~min}$ at each of the 5 exoskeleton stiffness conditions in a randomized order. We instrumented participants with surface electromyography on the left shank and motion capture markers on the lower limbs and pelvis.

\section{Steady state metabolic energy expenditure}

We collected indirect calorimetry data for each walking trial. To allow participants' metabolic rate to reach steady state, each walking trial lasted $5 \mathrm{~min}$. The order 
of the steady state metabolic conditions was rerandomized.

\section{Biomechanics measurements}

Lower-limb kinematics were measured using a reflective marker based motion capture system $(120 \mathrm{~Hz}$, Vicon, Oxford, UK) and participants were instrumented with 44 reflective markers to capture 6-DOF motion (3 rotational, 3 translational) of the foot, shank, thigh, and pelvis. Joint angles were calculated from marker data and joint angular velocities were calculated as the first derivative of the joint angle (Visual 3D, C-Motion, Germantown, MD). Ground reaction forces were captured with a split-belt instrumented treadmill $(960 \mathrm{~Hz})$. We performed inverse dynamics analysis to calculate the net joint moments about the ankle, knee, and hip. Analog data was filtered at $25 \mathrm{~Hz}$ and marker positions were filtered at $6 \mathrm{~Hz}$. The biological contribution to the total ankle joint moment was calculated by subtracting the measured exoskeleton torque from the total ankle joint moment. Joint angles and moments were reported for the sagittal plane. 6-DOF joint power with a rigid foot was calculated using techniques similar to Zelik et al. [34].

For a given participant, time-domain measurements for each of the 18 conditions were calculated by time normalizing each stride between heel-strike and heelstrike of the subsequent stride. Clean strides (average of $15.5 \pm 1.1$ strides) from the last $20 \mathrm{~s}$ of the walking bout were then averaged together to obtain a single normalized stride for a given condition. Integrated and peak values were calculated prior to intra-stride averaging. Average stride joint moments and powers were calculated by integrating the time-domain moment/power curves and then dividing by stride time. Peak values for a given measurement and condition are the average of the peaks for each stride within that condition. Average data for particular phases of a stride were computed as the time-integral over that phase divided by the time elapsed during that gait phase. To obtain the average moment per unit time (moment rate), we divided again by the stride/stance time over which the integral was taken to obtain the average moment rate $\left(\mathrm{Nm} \mathrm{kg}^{-1} \mathrm{~s}^{-1}\right)$. (Note: This rate represents an average moment per unit time and is not a measure of how rapidly the moment is generated).

\section{Electromyography (EMG) measurements}

Muscle activity of the ankle plantarflexors (medial (MG) and lateral (LG) gastrocnemius, soleus (SOL)), ankle dorsiflexors (tibialis anterior (TA)) as well as a hamstrings (biceps femoris long head (BFL)) and quadriceps muscle (rectus femoris (RF)) were measured with surface electromyography (EMG) on the left leg (SX230,
Biometrics, Newport, UK). To obtain a linear envelope, the raw EMG data was high-pass filtered at $20 \mathrm{~Hz}$, rectified, and low-pass filtered at $10 \mathrm{~Hz}$.

Integrated EMG (iEMG) was computed as the timeintegral of the EMG linear envelope averaged across each stride for a given condition. The amplitude of the EMG envelope and the integrated EMG for each muscle was normalized to the peak amplitude for that muscle observed across all conditions and speeds for each participant. Then, we divided the iEMG by the time period over which it was integrated to get the average EMG. Finally, to get average muscle activation per unit time, we again divided the average EMG by the time period over which it was averaged to obtain average muscle activation (EMG) rate $\left(\mathrm{s}^{-1}\right)$. (Note: This rate represents an average activation per unit time and is not a measure of how rapidly the muscle turns on and off.)

\section{Metabolic energy cost measurements}

We calculated metabolic power $\left(\mathrm{W} \mathrm{kg}^{-1}\right)$ using a portable indirect calorimetry system (OxyCon Mobile, Vyaire Medical, Mettawa, IL) and applied standard calorimetry equations [35]. To obtain net metabolic power for each condition, we subtracted metabolic power collected during the pretest standing trials from metabolic power for the walking conditions. In each trial, we averaged breath-by-breath data over the last minute of each fiveminute trial.

\section{Rate of average moment and rate of average muscle activation}

Inspired by the field of integrative physiology and research findings from Taylor, Kram, and colleagues [3642], we calculated the ankle moment and muscle activation summary statistics as a 'rate' which was the average of the metric per unit time (e.g. average moment per second, or average muscle activation per second). We used this approach because it provides improved understanding of the relationship between metabolic rate and neuromechanical outcomes (e.g., muscle activation or biological joint moment per unit time). Please see Supplementary Text of [43] for in-depth discussion.

\section{Statistics}

Statistical analysis was only performed on exoskeleton conditions in order to isolate the influence of exoskeleton stiffness from the effect of the added mass and other structural features of the device. For joint dynamics, EMG, and net metabolic power, we reported the means and standard error calculated across participants. Net metabolic rate was the primary outcome measure in this study. Based on our previous work [10], we expected a second-order (i.e., quadratic) relationship between net metabolic power $\left(\mathrm{W} \mathrm{kg}^{-1}\right)$ and exoskeleton stiffness 
$\left(\mathrm{Nm} \mathrm{rad}^{-1}\right)$ for which there would be a minimum at an intermediate stiffness. Therefore, we performed a threefactor, mixed-model ANOVA (random effect: participant; main effect: $k_{\text {exo }} k_{\text {exo }}^{2}$ ) at each walking speed to test the effect of exoskeleton stiffness on net metabolic power $(\alpha=0.05$; JMP Pro, SAS, Cary, NC). For the speeds where we found a significant main effect, we calculated the optimal stiffness as the minimum of the 2nd order regression. We then tested the two stiffness conditions bounding the minimum of the 2 nd order regression to determine if there was a significant reduction compared to no assistance $\left(\mathrm{k}_{\text {exo }}=0 \mathrm{Nm} \mathrm{rad}^{-1}\right)$ using post-hoc pairwise t-tests with Bonferroni correction. In a secondary analysis, for each walking speed, we performed a two-factor ANOVA (random: participant, main effect: $k_{\text {exo }}$ ) test to examine the relationship between users' joint biomechanics and muscle activity and ankle exoskeleton stiffness. We performed a number of within-participant, linear, least-squares regression (LLSR) analyses to test for relationships between changes in users' joint neuromechanics and changes in users' net metabolic rate with respect to the no assistance $\left(k_{\text {exo }}=0\right)$ condition. Finally, in a tertiary analysis, we performed a two-factor ANOVA to test for an effect of walking speed on users' joint biomechanics and muscle activity (random: participant, main effect: walking speed, $\mathrm{k}_{\text {exo }}$ ). A Shapiro-Wilk W test confirmed normality for all tests where relevant.

\section{Results}

\section{Metabolic cost}

Exoskeleton rotational stiffness reduced the metabolic cost of walking at 1.25 and $1.75 \mathrm{~m} \mathrm{~s}^{-1}$. At $1.5 \mathrm{~m} \mathrm{~s}^{-1}$, exoskeleton assistance resulted in increased metabolic cost for all conditions (Fig. 2). A three-factor, mixed-model ANOVA indicated a significant relationship between net metabolic rate and exoskeleton stiffness squared at both $1.25 \mathrm{~m} \mathrm{~s}^{-1}\left(n=11, \mathrm{k}_{\text {exo }}^{2} p=0.022\right.$; Net Metabolic Rate $=$ $3.096-0.001 * \mathrm{k}_{\text {exo }}+7.047 * 10^{-6 *} \mathrm{k}_{\text {exo }}^{2}$ ) and $1.75 \mathrm{~m} \mathrm{~s}^{-1}$ $\left(\mathrm{k}_{\text {exo }}^{2} p=0.009\right.$; Net Metabolic Rate $=5.578-0.002$ * $\mathrm{k}_{\text {exo }}+1.318 * 10^{-5} * \mathrm{k}_{\text {exo }}^{2}$ ). We found no significant relationship between exoskeleton stiffness and net metabolic rate for the intermediate walking speed of $1.5 \mathrm{~m} \mathrm{~s}^{-1}$. $\left(\mathrm{k}^{2}{ }_{\text {exo }}, p=0.71\right)$. The optimal exoskeleton stiffness, calculated as the minimum of the 2nd order regression model, was $70 \mathrm{Nm} \mathrm{rad}^{-1}$ for slow walking $\left(1.25 \mathrm{~m} \mathrm{~s}^{-1}\right)$ and $79 \mathrm{Nm} \mathrm{rad}^{-1}$ for fast walking $\left(1.75 \mathrm{~m} \mathrm{~s}^{-1}\right)$ conditions. We found no optimal exoskeleton stiffness for intermediate speed $\left(1.50 \mathrm{~m} \mathrm{~s}^{-1}\right)$ walking. For walking speeds of $1.25 \mathrm{~m} \mathrm{~s}^{-1}$ and $1.75 \mathrm{~m} \mathrm{~s}^{-1}$, we further analyzed the two stiffness conditions bounding the minimum of the 2nd order regression $\left(50 \mathrm{Nm} \mathrm{rad}^{-1}\right.$ and $100 \mathrm{Nm}$ $\mathrm{rad}^{-1}$ for both speeds) to determine if there was a significant reduction compared to no assistance $\left(k_{\text {exo }}=0\right.$

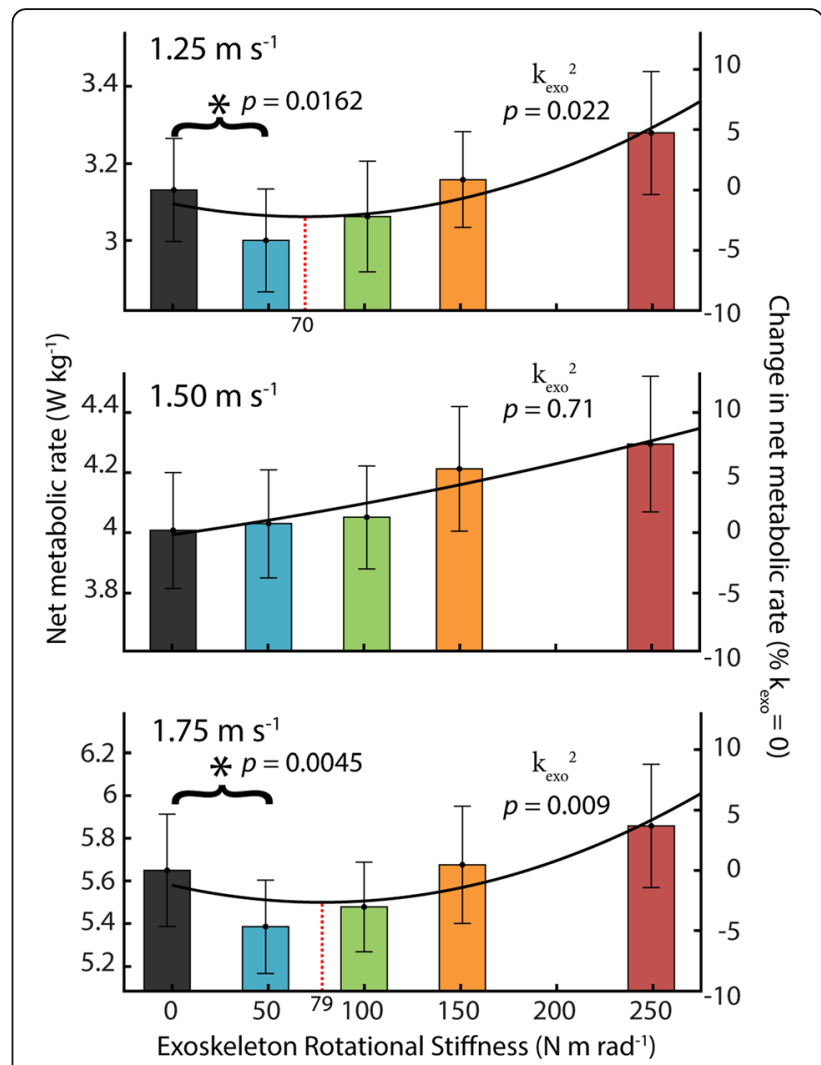

Fig. 2 Net metabolic rate across stiffness and speed. Steady state metabolic cost for five exoskeleton stiffness conditions ( $k_{\text {exo }}=0,50$, 100, 150, $\left.250 \mathrm{Nm} \mathrm{rad}^{-1}\right)$ and three walking speeds $(1.25,1,50,1.75 \mathrm{~m}$ $\left.\mathrm{s}^{-1}\right)$. A significant relationship was found between exoskeleton rotational stiffness and net metabolic rate at slow and fast walking speeds ( $n=11$; mixed model ANOVA with second order term $k_{\text {exoi }}^{2}$ $1.25 \mathrm{~m} \mathrm{~s}^{-1} \mathrm{p}_{\text {stifffness }}^{2}=0.022 ; 1.75 \mathrm{~m} \mathrm{~s}^{-1} \mathrm{p}_{\text {stifffness }}^{2}=0.009$ ). No significant relationship was observed for the intermediate $1.50 \mathrm{~m} \mathrm{~s}^{-1}$ speed. The lowest stiffness $\left(50 \mathrm{Nm} \mathrm{rad}^{-1}\right)$ resulted in metabolic reductions of $4.2 \pm$ $1.7 \%$ (mean \pm s.e.m) at $1.25 \mathrm{~m} \mathrm{~s}^{-1}$ and $4.7 \pm 1.3 \%$ at $1.75 \mathrm{~m} \mathrm{~s}^{-1}\left({ }^{*}=\right.$ paired t-test $a=0.025$ ). The solid line is a quadratic best-fit curve where the stiffness at the minima is indicated by the vertical dashed line

$\mathrm{Nm} \mathrm{rad).} \mathrm{At} 1.25 \mathrm{~m} \mathrm{~s}^{-1}$, we measured a significant reduction in net metabolic rate between the $\mathrm{k}_{\text {exo }}=0$ and $50 \mathrm{Nm} \mathrm{rad}^{-1}$ conditions $(-4.2 \% \mathrm{CI}<-0.4,-7.8 \%>$; onetailed paired t-test $p=0.0162 ; \alpha=0.025)$ but not 100 $\mathrm{Nm} \mathrm{rad}^{-1}(-2.3 \% \mathrm{CI}<1.6,-6.2 \%>$; one-tailed paired $\mathrm{t}$ test $p=0.108 ; \alpha=0.025)$. At $1.75 \mathrm{~m} \mathrm{~s}^{-1}$, we measured a significant reduction in net metabolic rate between the $\mathrm{k}_{\text {exo }}=0$ and $50 \mathrm{Nm} \mathrm{rad}^{-1}$ conditions $(-4.7 \% \mathrm{CI}<-1.4$, $7.1 \%>$; one-tailed paired t-test $p=0.0045 ; \alpha=0.025)$ but not $100 \mathrm{Nm} \mathrm{rad}^{-1}(-2.6 \% \mathrm{CI}<1.1,-6.2 \%>$; one-tailed paired t-test $p=0.0551 ; \alpha=0.025)$. Net metabolic power results for individual participants are reported in Supplemental Table 1.

To highlight the effect of exoskeleton stiffness on biomechanics and walking economy, the results focuses primarily on group effects and on the effect of two 
exoskeleton stiffnesses with respect to the $0 \mathrm{Nm} \mathrm{rad}^{-1}$ (no assistance) condition. The $50 \mathrm{Nm} \mathrm{rad}^{-1}$ condition details what occurs with a stiffness that is low but results in the reduction in metabolic rate for slow and fast walking. The $250 \mathrm{Nm} \mathrm{rad}^{-1}$ condition details what occurs at a perceptually very stiff condition which also resulted in the metabolic maximum.

\section{Gait neuromechanics at $1.25 \mathrm{~m} \mathrm{~s}^{-1}$}

With increasing exoskeleton stiffness, the total ankle (exoskeleton torque + biological moment) average moment rate over the stride increased $(p<0.0001)$ as exoskeleton average torque rate over the stride increased $(p<0.0001)$ and despite a decrease in biological ankle average moment rate over the stride $(p<0.0001)$ (Fig. 3a, $\mathrm{Ai}$, Supp. Figure 1). (Note: This rate represents an average moment per unit time and is not a measure of how rapidly the moment is generated). Summary statistics are reported in Supplementary Table 2. Compared to $0 \mathrm{Nm}$ $\mathrm{rad}^{-1}, 50 \mathrm{Nm} \mathrm{rad}^{-1}$ resulted in a $12 \%$ reduction in average biological moment rate over the stride and $250 \mathrm{Nm}$ $\mathrm{rad}^{-1}$ resulted in a $19 \%$ reduction. In early stance $(0-$ $40 \%)$, the total ankle average moment rate increased $(p<0.0001)$ as exoskeleton average torque rate increased $(p<0.0001)$ while biological average moment rate decreased only slightly $(p=0.0285)$. For $250 \mathrm{Nm} \mathrm{rad}^{-1}$ relative to $0 \mathrm{Nm} \mathrm{rad}^{-1}$, the total ankle average moment rate in early stance increased by $52 \%$ as the exoskeleton produced $0.49 \pm 0.06 \mathrm{Nm} \mathrm{kg}^{-1} \mathrm{~s}^{-1}$ (mean \pm s.e.m) of torque and biological moment decreased only slightly (Fig. 3Aii). At the time of peak moment, the total ankle joint moment remained constant as exoskeleton torque increased $(p<0.0001)$ and biological moment decreased $(p<$ 0.0001 ) (Fig. 3Aiii). Compared to $0 \mathrm{Nm} \mathrm{rad}^{-1}, 50 \mathrm{Nm}$ $\mathrm{rad}^{-1}$ resulted in a $12 \%$ reduction in peak biological moment and the $250 \mathrm{Nm} \mathrm{rad}^{-1}$ resulted in a $29 \%$ reduction.

Average positive mechanical power of the ankle declined with increasing stiffness $(p<0.0001)$, by $30 \%$ at $250 \mathrm{Nm} \mathrm{rad}^{-1}$ relative to $0 \mathrm{Nm} \mathrm{rad}^{-1}$ (Fig. 3b, Bi). Negative mechanical power also declined $(p<0.0001)$ (Fig. 3Bii). Exoskeleton average mechanical positive power increased with initial increase in stiffness to $0.036 \pm 0.003 \mathrm{~W} \mathrm{~kg}^{-1}$ at $50 \mathrm{Nm} \mathrm{rad}^{-1}$ but was roughly the same at $0.064 \mathrm{~W} \mathrm{~kg}^{-1}$ for the $100 \mathrm{Nm} \mathrm{rad}^{-1}$ through $250 \mathrm{Nm} \mathrm{rad}^{-1}$ conditions.

At $1.25 \mathrm{~m} \mathrm{~s}^{-1}$, SOL activation rate decreased by $9 \%$ for $50 \mathrm{Nm} \mathrm{rad}^{-1}, 13 \%$ at $100 \mathrm{Nm} \mathrm{rad}^{-1}$, and $11 \%$ at $250 \mathrm{Nm}$ $\mathrm{rad}^{-1}$ compared to $0 \mathrm{Nm} \mathrm{rad}^{-1}$, but the linear effect of stiffness on stride average SOL activation rate was not significant (Fig. 3c Ci). Stride average TA activation rate increased with stiffness $(p<0.0001)$ (Fig. 3Cii). The activation rate of non-weighted sum of stride average SOL and TA $(\mathrm{SOL}+\mathrm{TA})$ was reduced by $5 \%$ at $50 \mathrm{Nm} \mathrm{\textrm {rad } ^ { - 1 }}$ and increased by $10 \%$ at $250 \mathrm{Nm} \mathrm{rad}^{-1}$ but the linear effect of stiffness was not significant (Fig. 3Ciii). For LG and MG, we measured an increase in stride average activation rate with increasing stiffness $(p=0.0003, p=$ 0.001 ) which was up $22 \%$ at $250 \mathrm{Nm} \mathrm{rad}^{-1}$ relative to 0 $\mathrm{Nm} \mathrm{rad}^{-1}$ (Supp. Figure 2B, C). Stride average muscle activation is reported in Supplemental Table 2.

Peak dorsiflexion angle decreased with increasing stiffness $(p<0.0001)$. Angle decreased from $17.8 \pm 1.24$ degrees at $0 \mathrm{Nm} \mathrm{rad}^{-1}$ to $15.0 \pm 1.26$ degrees at $50 \mathrm{Nm}$ $\mathrm{rad}^{-1}$ and $8.12 \pm 1.39$ degrees at $250 \mathrm{Nm} \mathrm{rad}^{-1}$ (Fig. 3d, Di). From 0 to $250 \mathrm{Nm} \mathrm{rad}^{-1}$, stride time decreased ( $p=$ 0.0063 ) from $1.09 \pm 0.02 \mathrm{~s}$ to $1.04 \pm 0.04 \mathrm{~s}$, stance time decreased $(p=0.0002)$ from $0.70 \pm 0.01 \mathrm{~s}$ to $0.66 \pm 0.02 \mathrm{~s}$, and the ratio of stance time to stride time (i.e. duty factor $)$ decreased $(p=0.0312)$ from $64.55 \pm 0.25 \%$ to $63.82 \pm 0.26 \%$ (Supp. Table 2).

At more proximal joints, increasing exoskeleton stiffness resulted in a shift from knee extension to knee flexion (Supp. Figure 3). Average extension moment rate decreased $(p<0.0001)$ from 0.18 to $0.08 \mathrm{Nm} \mathrm{kg}^{-1} \mathrm{~s}^{-1}$ and average flexion moment rate increased from 0.16 to $0.40 \mathrm{Nm} \mathrm{kg}^{-1}(p<0.0001)$ across the range of exoskeleton stiffness (Supp. Figure 3B). Knee average negative and net mechanical power increased as exoskeleton stiffness increased $(p<0.0001)$ (Supp. Figure 3C). Hip flexion $(p=0.003)$ and extension $(p=0.0002)$ moment rate increased, and average positive $(p=0.0387)$ and net $(p=0.0447)$ mechanical power increased with stiffness (Supp. Figure $4 \mathrm{~B}, \mathrm{C}$ ). The stance average activation rate of the BFL increased $(p<0.0001)$, by $66 \%$ at $250 \mathrm{Nm}$ $\mathrm{rad}^{-1}$, and stride average RF activation rate increased by $28 \%$ in the stiffest condition though group effect was not significant $(p=0.0646)$ (Supp. Figure 2E, F).

\section{Gait neuromechanics at $1.50 \mathrm{~m} \mathrm{~s}^{-1}$}

With increasing exoskeleton stiffness, the total ankle average moment rate over the stride increased $(p<$ $0.0001)$ as exoskeleton average torque rate over the stride increased $(p<0.0001)$ despite a decrease in biological ankle average moment rate over the stride $(p<$ 0.0001) (Fig. 4a, Ai). Summary statistics are reported in Supplementary Table 3. Compared to $0 \mathrm{Nm} \mathrm{rad}^{-1}, 50$ $\mathrm{Nm} \mathrm{rad}^{-1}$ resulted in a $9 \%$ reduction in average biological moment rate over the stride and $250 \mathrm{Nm} \mathrm{rad}^{-1}$ resulted in a $15 \%$ reduction. In early stance $(0-40 \%)$, the total ankle average moment rate increased $(p<0.0001)$ as exoskeleton average torque rate increased $(p<0.0001)$ while biological average moment rate remained constant. For the $250 \mathrm{Nm} \mathrm{rad}^{-1}$ compared to $0 \mathrm{Nm} \mathrm{rad}^{-1}$, the total ankle average moment rate in early stance increased by $46 \%$ as the exoskeleton produced $0.52 \pm 0.06 \mathrm{Nm} \mathrm{kg}^{-1}$ $\mathrm{s}^{-1}$ of torque and biological moment did not change (Fig. 4Aii). At the time of peak moment, the total ankle joint moment remained constant as exoskeleton torque 


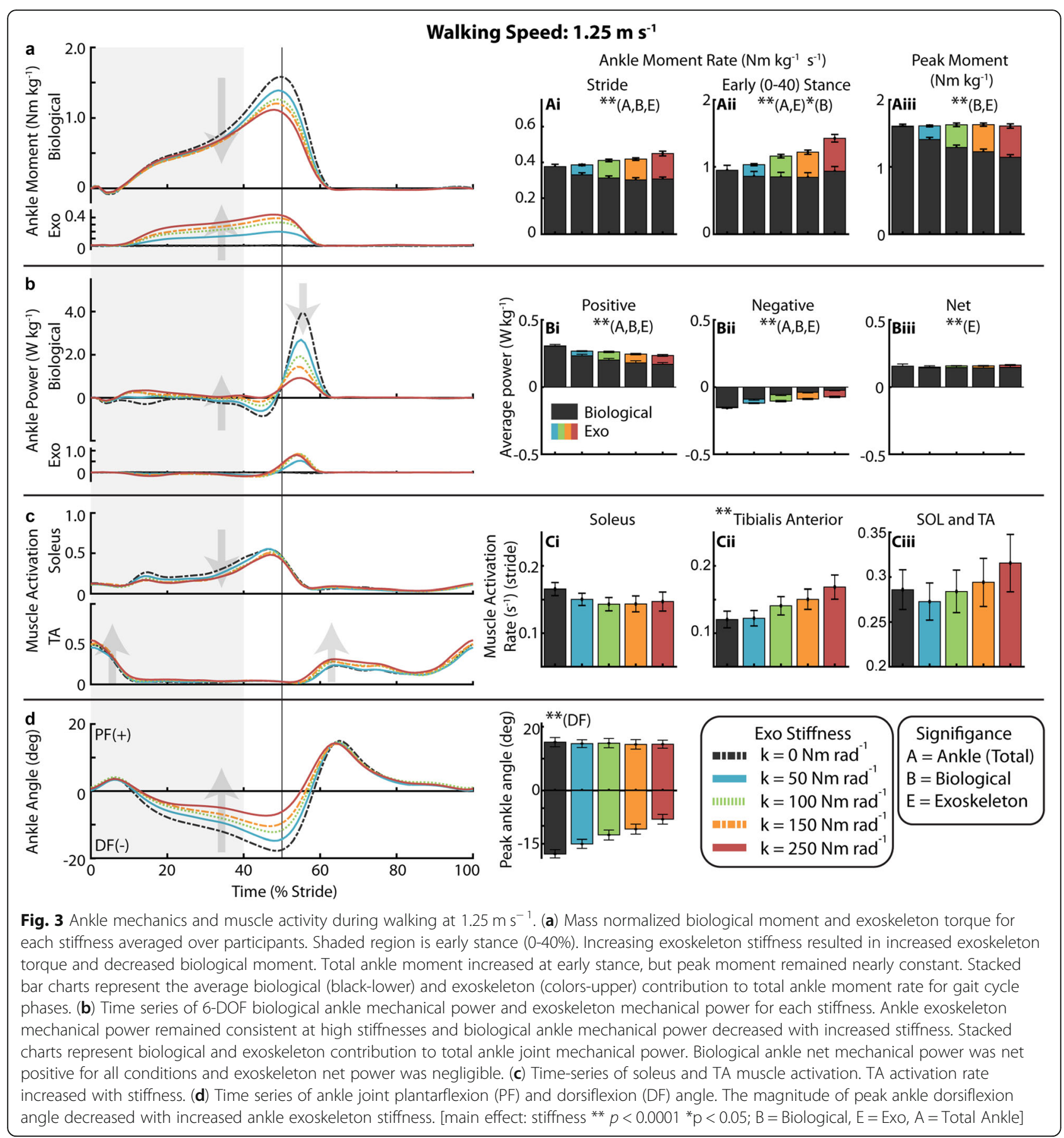

increased $(p<0.0001)$ and biological moment decreased $(p<0.0001)$ (Fig. 4Aiii). Compared to $0 \mathrm{Nm} \mathrm{rad}^{-1}, 50$ $\mathrm{Nm} \mathrm{rad}^{-1}$ resulted in a $9 \%$ reduction in peak biological moment and $250 \mathrm{Nm} \mathrm{rad}^{-1}$ resulted in a $23 \%$ reduction.

Average positive mechanical power of the ankle declined with increasing stiffness $(p<0.0001)$, by $21 \%$ at $250 \mathrm{Nm} \mathrm{rad}^{-1}$ relative to $0 \mathrm{Nm} \mathrm{rad}^{-1}$ (Fig. 4b, Bi). Negative mechanical power also declined $(p<0.0001)$ (Fig. 4Bii). Exoskeleton average mechanical positive power increased with initial increase in stiffness to $0.037 \pm 0.004$
W kg-1 at $50 \mathrm{Nm} \mathrm{rad}^{-1}$ but was roughly the same at $0.058 \mathrm{~W} \mathrm{~kg}^{-1}$ for the $100 \mathrm{Nm} \mathrm{rad}^{-1}$ through $250 \mathrm{Nm}$ $\mathrm{rad}^{-1}$ conditions.

Compared to $0 \mathrm{Nm} \mathrm{rad}^{-1}$, SOL activation rate decreased by $7 \%$ for $50 \mathrm{Nm} \mathrm{rad}^{-1}$ and by only $4 \%$ at 250 $\mathrm{Nm} \mathrm{rad}{ }^{-1}$ and the linear effect of stiffness was not significant. (Fig. 4c,Ci). Stride average TA activation rate increased with stiffness $(p=0.0032)$ (Fig. 4Cii). The $\mathrm{SOL}+\mathrm{TA}$ activation rate increased for all stiffnesses (Fig. 4Ciii) though not significantly $(p=0.069)$. Compared to 


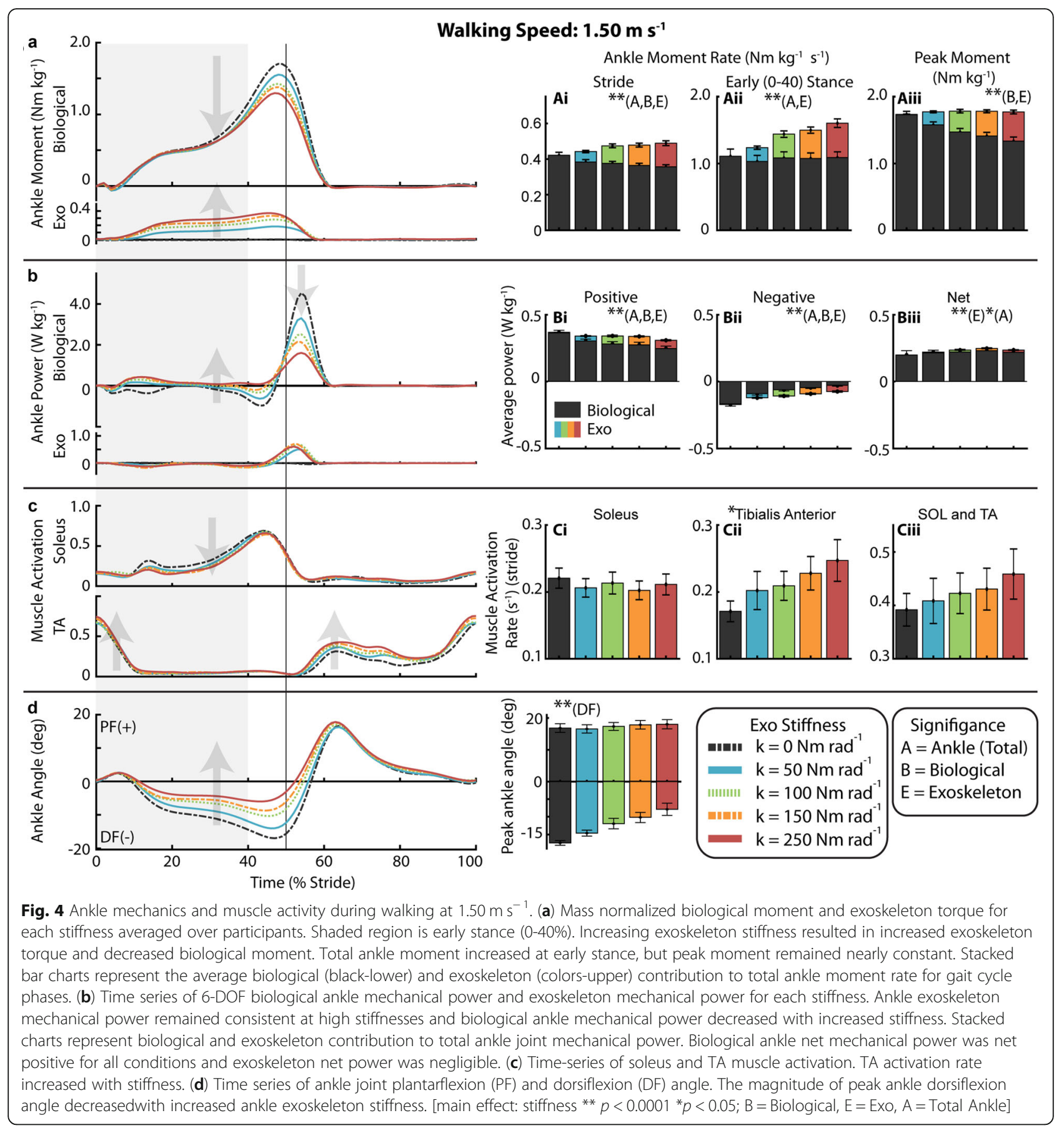

$0 \mathrm{Nm} \mathrm{rad}{ }^{-1}$, SOL+TA activation rate increased by $4 \%$ at $50 \mathrm{Nm} \mathrm{rad}^{-1}$ and $17 \%$ at $250 \mathrm{Nm} \mathrm{rad}^{-1}$. For the LG and MG, we measured an average increase in stride average activation rate $(p<0.0001)$ which was up $21 \%$ at 250 $\mathrm{Nm} \mathrm{rad}^{-1}$ relative to $0 \mathrm{Nm} \mathrm{rad}^{-1}$ (Supp. Figure 2B, C). Stride average muscle activation is reported in Supplemental Table 3.

Peak dorsiflexion angle decreased $(p<0.0001)$ from $17.4 \pm 0.7$ at $0 \mathrm{Nm} \mathrm{rad}^{-1}$ to $14.6 \pm 0.9$ degrees at $50 \mathrm{Nm}$ $\mathrm{rad}^{-1}$ and to $7.9 \pm 1.7$ degrees at $250 \mathrm{Nm} \mathrm{rad}^{-1}$ (Fig. $4 \mathrm{~d}$,
Di). From 0 to $250 \mathrm{Nm} \mathrm{rad}^{-1}$, stride time decreased ( $p=$ 0.0010 ) from $1.00 \pm 0.02 \mathrm{~s}$ to $0.98 \pm 0.02 \mathrm{~s}$, stance time decreased $(p<0.0001)$ from $0.63 \pm 0.01 \mathrm{~s}$ to $0.61 \pm 0.02 \mathrm{~s}$, and the ratio of stance time to stride time decreased $(p=$ 0.0008 ) from $63.1 \pm 0.2 \%$ to $62.5 \pm 0.3 \%$ (Supp. Table 3 ).

At more proximal joints, increasing exoskeleton stiffness resulted in a shift from knee extension to knee flexion for both speeds $(p<0.0001)$ and shift to more negative joint mechanical power $(p<0.0001)$ (Supp. Figure 3). At the hip, there was a slight increase in flexion 
moment with ankle exoskeleton stiffness $(p=0.011)$ (Supp. Figure 4). The stance average activation rate of the BFL increased $(p<0.0001)$, by $63 \%$ at $250 \mathrm{Nm} \mathrm{rad}^{-1}$, and stride average RF activation rate increased by $23 \%$ at $250 \mathrm{Nm} \mathrm{rad}{ }^{-1}$ though group effect was not significant $(p=0.0594)$ (Supp. Figure 2E, F).

\section{Gait neuromechanics at $1.75 \mathrm{~m} \mathrm{~s}^{-1}$}

With increasing exoskeleton stiffness, the total ankle average moment rate over the stride increased $(p<$ $0.0001)$ as exoskeleton average torque rate over the stride increased $(p<0.0001)$ despite a decrease in biological ankle average moment rate over the stride $(p<$ 0.0001) (Fig. 5a, Ai). Summary statistics are reported in Supplementary Table 4. Compared to $0 \mathrm{Nm} \mathrm{rad}^{-1}, 50$ $\mathrm{Nm} \mathrm{rad}^{-1}$ resulted in a $7 \%$ reduction in average biological moment rate over the stride and $250 \mathrm{Nm} \mathrm{rad}^{-1}$ resulted in a $13 \%$ reduction. In early stance $(0-40 \%)$, the total ankle average moment rate increased $(p<0.0001)$ as exoskeleton average torque rate increased $(p<0.0001)$ while biological average moment remained constant. For $250 \mathrm{Nm} \mathrm{rad}^{-1}$ relative to $0 \mathrm{Nm} \mathrm{rad}^{-1}$, the total ankle average moment rate in early stance increased by $58 \%$ as the exoskeleton produced $0.56 \pm 0.07 \mathrm{Nm} \mathrm{kg}^{-1} \mathrm{~s}^{-1}$ of torque and biological moment did not change (Fig. 5aii). At the time of peak moment, the total ankle joint moment remained constant as exoskeleton torque increased $(p<0.0001)$ and biological moment decreased $(p<$ 0.0001) (Fig. 5Aiii). Compared to $0 \mathrm{Nm} \mathrm{rad}^{-1}, 50 \mathrm{Nm}$ $\mathrm{rad}^{-1}$ resulted in a $9 \%$ reduction in peak biological moment and the $250 \mathrm{Nm} \mathrm{rad}^{-1}$ resulted in a $22 \%$ reduction.

Average positive mechanical power of the ankle declined with increasing stiffness $(p<0.0001)$, by $21 \%$ at $250 \mathrm{Nm} \mathrm{rad}^{-1}$ relative to $0 \mathrm{Nm} \mathrm{rad}^{-1}$ (Fig. $5 \mathrm{~b}, \mathrm{Bi}$ ). Negative mechanical power also declined $(p<0.0001)$ (Fig. 5 Bii). Exoskeleton average mechanical positive power increased with initial increase in stiffness to $0.041 \pm 0.007$ $\mathrm{W} \mathrm{kg}-1$ at $50 \mathrm{Nm} \mathrm{rad}^{-1}$ but was again roughly the same at $0.064 \mathrm{~W} \mathrm{~kg}^{-1}$ for the $100 \mathrm{Nm} \mathrm{rad}^{-1}$ through $250 \mathrm{Nm}$ $\mathrm{rad}^{-1}$ conditions.

At $1.75 \mathrm{~m} \mathrm{~s}^{-1}$, the stride average SOL activation rate decreased by $6 \%$ for the $50 \mathrm{Nm} \mathrm{rad}^{-1}, 9 \%$ at the $100 \mathrm{Nm}$ $\mathrm{rad}^{-1}$, and $2 \%$ at $250 \mathrm{Nm} \mathrm{rad}^{-1}$ compared to $0 \mathrm{Nm}$ $\mathrm{rad}^{-1}$, but the linear effect of stiffness was not significant (Fig. $5 \mathrm{c}, \mathrm{Ci}$ ). Stride average TA activation rate increased with stiffness $(p<0.0001)$ (Fig. 5 Cii). The SOL+TA activation rate appeared to be minimized at $100 \mathrm{Nm} \mathrm{rad}^{-1}$ (Fig. 5Ciii) but the linear effect of stiffness on SOL+TA activation was not significant. Compared to $0 \mathrm{Nm} \mathrm{rad}^{-1}$, SOL+TA activation rate was reduced by $2 \%$ at the 50 $\mathrm{Nm} \mathrm{rad}^{-1}, 3 \%$ at $100 \mathrm{Nm} \mathrm{rad}^{-1}$, and increased by $8 \%$ at $250 \mathrm{Nm} \mathrm{rad}^{-1}$. For the lateral (LG) and medial gastrocnemius (MG) muscles, we measured an average increase in stride average activation rate $(p=0.0006, p<$
0.0001 ) which was up $27 \%$ at $250 \mathrm{Nm} \mathrm{rad}^{-1}$ relative to 0 $\mathrm{Nm} \mathrm{rad}^{-1}$ (Supp. Figure 2B, C). Stride average muscle activation is reported in Supplemental Table 4.

Peak dorsiflexion angle decreased with increasing stiffness $(p<0.0001)$ from $16.5 \pm 1.01$ degrees at $0 \mathrm{Nm} \mathrm{rad}^{-1}$ to $13.7 \pm 1.1$ degrees at $50 \mathrm{Nm} \mathrm{rad}^{-1}$ and $6.61 \pm 1.56$ degrees at $250 \mathrm{Nm} \mathrm{rad}^{-1}$ (Fig. $5 \mathrm{~d}, \mathrm{Di}$ ). Peak plantarflexion angle increased with increasing stiffness $(p=0.0116)$ from $16.5 \pm 1.41$ degrees at $0 \mathrm{Nm} \mathrm{rad}^{-1}$ to $17.61 \pm 1.39$ degrees at $50 \mathrm{Nm} \mathrm{rad}^{-1}$ and $19.65 \pm 1.21$ degrees at 250 $\mathrm{Nm} \mathrm{rad}^{-1}$ (Fig. 5Di). From 0 to $250 \mathrm{Nm} \mathrm{rad}^{-1}$, stride time decreased $(p=0.0115)$ from $0.93 \pm 0.02 \mathrm{~s}$ to $0.91 \pm 0.02 \mathrm{~s}$, stance time decreased $(p=0.0044)$ from $0.58 \pm 0.01 \mathrm{~s}$ to $0.56 \pm 0.02 \mathrm{~s}$, and ratio of stance time to stride time did not significantly decrease from $62.3 \% \pm$ 0.24\% (Supp. Table 4).

At the knee, increasing exoskeleton stiffness again resulted in a shift from knee extension to knee flexion $(p<0.0001)$ (Supp. Figure 3B) and shift to more negative joint mechanical power $(p<0.0001)$ (Supp. Figure $3 C)$. The stance average activation rate of the BFL increased $(p<0.0001)$, by $56 \%$ at $250 \mathrm{Nm} \mathrm{rad}^{-1}$, and stride average $\mathrm{RF}$ activation rate increased by $19 \%$ at $250 \mathrm{Nm} \mathrm{rad}^{-1}$ though group effect was not significant $(p=0.0715)$ (Supp. Figure 2E, F).

\section{Comparison across speeds}

The relative contribution of the exoskeleton to total ankle joint mechanics reduced as walking speed increased. With increasing speed, peak total ankle moment $(p<0.0001)$ and peak biological ankle moment increased $(p<0.0001)$ but peak exoskeleton torque decreased $(p<$ $0.01)$ (Supp. Figure 1). From the slowest $\left(1.25 \mathrm{~ms}^{-1}\right)$ to the fastest $\left(1.75 \mathrm{~ms}^{-1}\right)$ speeds, when a stiffness of 250 $\mathrm{Nm} \mathrm{rad}^{-1}$ was applied the peak total ankle moment increased by $12 \%$ and peak biological ankle moment increased by $26 \%$ yet peak exoskeleton torque decreased by $23 \%$. Total positive mechanical power at the ankle increased with speed $(p<0.0001)$ despite little increase in exoskeleton positive mechanical power $(0.5 \%)$. Only at $50 \mathrm{Nm} \mathrm{rad}{ }^{-1}$ did we measure an increase in exoskeleton positive mechanical power (16\%) with increased walking speed.

From slow to fast walking, the ankle dorsiflexion angle decreased $(p<0.01)$ for all exoskeleton stiffness conditions, with an average decrease in peak dorsiflexion angle of 25\% (Supp. Figure 1). On the other hand, for all exoskeleton stiffness conditions, peak ankle plantarflexion angle increased with increasing walking speed $(p<$ $0.0001)$. Stride time decreased $(p<0.0001)$ by $0.15 \mathrm{~s}$ $(14 \%)$ on average from slow to fast speed and stance time decreased $(p<0.0001)$ by $0.12 \mathrm{~s}(17 \%)$ from slow to fast. The ratio of stance time to stride time decreased 


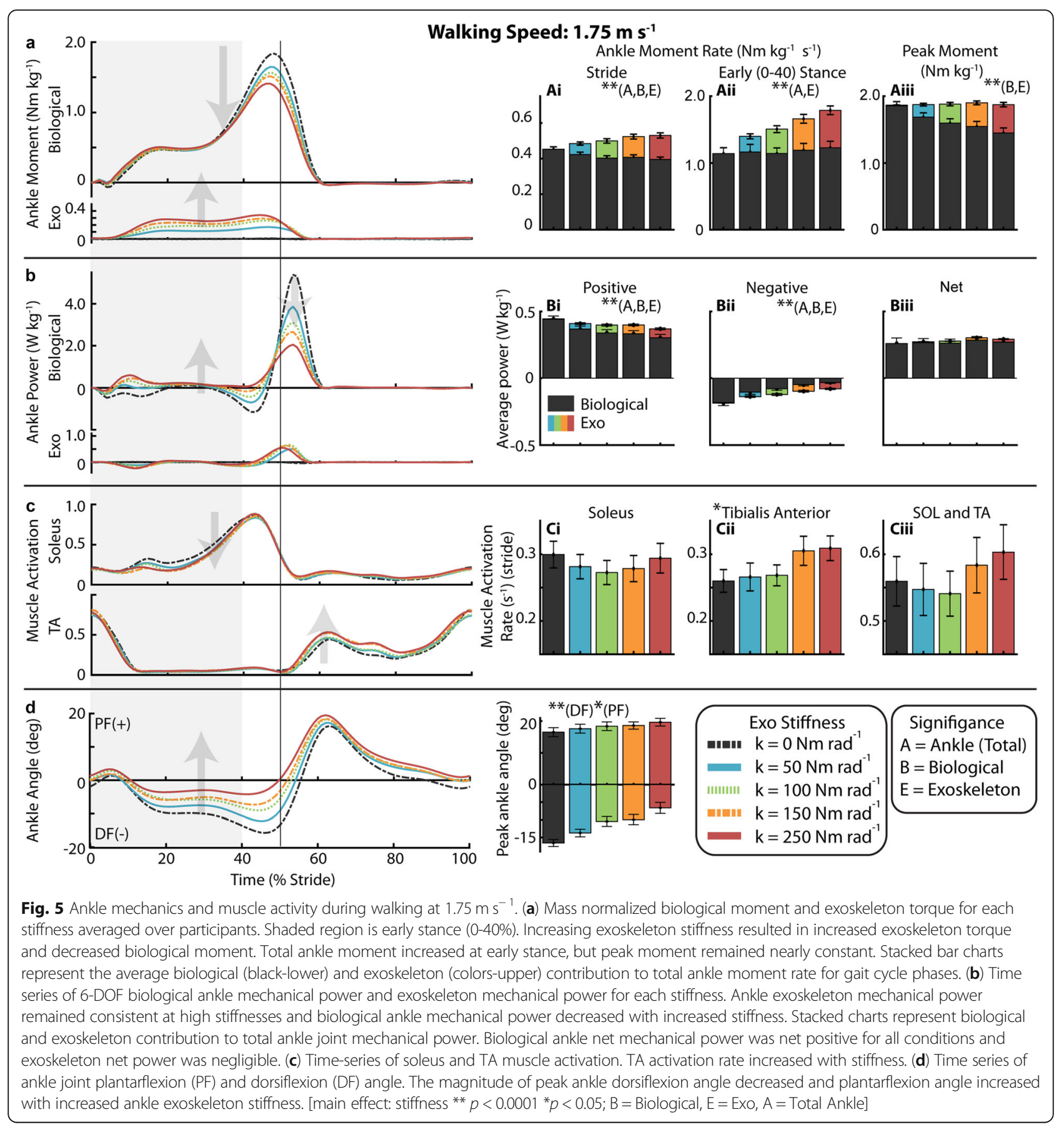

$(p<0.0001)$ from 64.2 to $61.8 \%$ of the gait cycle from slow to fast walking speed (Supp. Table 2-4).

Association between changes in users' joint mechanical/ muscle activation rates and changes in net metabolic rate Changes in users' SOL+TA muscle activation rate due to exoskeleton assistance were significantly correlated with measured changes in users' net metabolic rate (Fig. 6). This relationship held for the slow $\left(1.25 \mathrm{~m} \mathrm{~s}^{-1}: p=0.0009\right.$, $\left.\mathrm{R}^{2}=0.56\right)$, intermediate $\left(1.50 \mathrm{~m} \mathrm{~s}^{-1:} p=0.0497, \mathrm{R}^{2}=0.64\right)$, and fast walking speed $\left(1.75 \mathrm{~m} \mathrm{~s}^{-1:} p=0.0002, \mathrm{R}^{2}=0.69\right)$. Changes in SOL+TA activation rate were more highly correlated with changes in net metabolic rate when compared with measured changes in biological moment rate, biological power (i.e., mechanical work rate), or exoskeleton power (Fig. 6 and Table 1).

\section{Training}

Results from the training day indicate that participants adapted in a metabolically beneficial manner only in the 


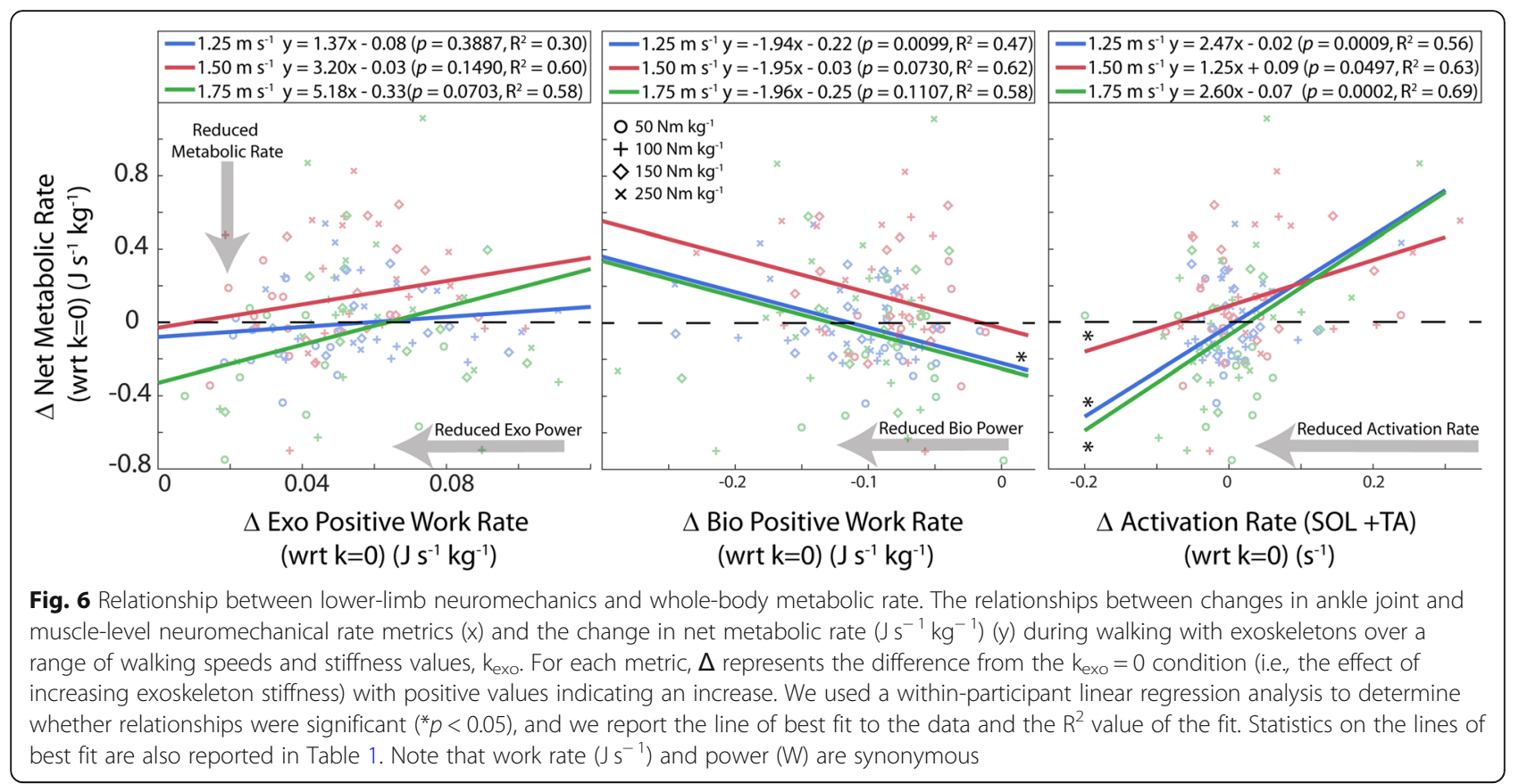

$50 \mathrm{Nm} \mathrm{rad}^{-1}$ exoskeleton stiffness condition (Supp. Figure 5). Over the training period, for $50 \mathrm{Nm} \mathrm{rad}^{-1}$ participants on average reduced their net metabolic power by 6.7\% ( $p=0.017$, two-tailed paired t-test). Two testing sessions later, the net metabolic rate during the steady-state testing day was on average $2.9 \%$ lower than the rate at the end of the training day though not significantly so $(p=0.6$, two-tailed paired t-test). Conversely, we measured an increase of $1.7 \%$ in net metabolic rate over the testing period for the three stiffest exoskeleton conditions. Values recorded during the steady-state metabolic data collection sessions were, on average, $7.8 \%$ lower for the three high stiffness conditions when compared to the training day.

\section{Discussion}

Our data suggest that passive elastic ankle exoskeletons can reduce metabolic cost at multiple, but not all, walking speeds (Fig. 2). The lowest tested exoskeleton rotational stiffness condition $\left(50 \mathrm{Nm} \mathrm{rad}^{-1}\right)$ resulted in a metabolic reduction of $-4.2 \%$ at slow $\left(1.25 \mathrm{~m} \mathrm{~s}^{-1}\right)$ and -
$4.7 \%$ at fast $\left(1.75 \mathrm{~m} \mathrm{~s}^{-1}\right)$ walking speeds when compared to no assistance (i.e. $\mathrm{k}_{\text {exo }}=0 \mathrm{Nm} \mathrm{rad}^{-1}=$ zero-torque). The results from this study for walking at $1.25 \mathrm{~m} \mathrm{~s}^{-1}$ support findings from our previous study where rotational stiffness was applied using physical springs rather than the emulated elastic system we present here [10]. At slow and fast walking speeds, we confirm a second order relationship $\left(\mathrm{k}_{\text {exo }}^{2}\right)$ between exoskeleton rotational stiffness and the users' net metabolic power, indicating a 'sweet-spot' such that balancing a trade-off between very high stiffness (not too stiff) and very low stiffness (not too compliant) is necessary to achieve metabolic benefit. Surprisingly, no exoskeleton stiffness condition resulted in an average decrease in metabolic cost at the intermediate speed $\left(1.5 \mathrm{~m} \mathrm{~s}^{-1}\right)$. Rather, for walking at $1.5 \mathrm{~m}$ $\mathrm{s}^{-1}$, we observed a monotonic linear increase in users' net metabolic rate with increasing exoskeleton stiffness.

Using our extensive set of human neuromechanical data across a range of functionally relevant walking speeds, we endeavored to gain a comprehensive

Table 1 Relationship between lower-limb joint and muscle-level neuromechanics and whole-body metabolic rate

\begin{tabular}{llll}
\hline Gait Cycle Phase & $1.25 \mathrm{~m} \mathrm{~s}^{-1}$ & $1.50 \mathrm{~m} \mathrm{~s}^{-1}$ & $1.75 \mathrm{~m} \mathrm{~s}^{-1}$ \\
\hline$\Delta$ Exoskeleton Positive Work Rate $\left(\mathrm{J} \mathrm{kg}^{-1} \mathrm{~s}^{-1}\right)$ & $p=0.3887$ & $p=0.1490$ & $p=0.0703$ \\
$\Delta$ Biological Positive Work Rate $\left(\mathrm{Jg}^{-1} \mathrm{~s}^{-1}\right)$ & $\boldsymbol{p}=\mathbf{0 . 0 0 9 9}, \mathbf{R}^{\mathbf{2}}=\mathbf{0 . 4 7}$ & $p=0.0730$ & $p=0.1107$ \\
$\Delta$ Biological Moment Rate $\left(\mathrm{N} \mathrm{kg}^{-1} \mathrm{~s}^{-1}\right)$ & $p=0.6574$ & $p=0.0502$ & $p=0.1273$ \\
$\Delta$ Soleus + Tibialis Anterior Activation Rate (unitless s & \\
\hline
\end{tabular}

The relationships between changes in ankle joint and muscle-level neuromechanical rate metrics $(\mathrm{x})$ and the change in net metabolic rate $\left(\mathrm{W} \mathrm{kg}{ }^{-1}\right)(\mathrm{y})$ during walking with exoskeletons over a range of walking speeds and stiffness values, $k_{\text {exo }}$. For each metric, $\Delta$ represents the difference from the $k_{\text {exo }}=0$ condition (i.e., the effect of increasing exoskeleton stiffness) with positive values indicating an increase. We used a within-participant linear regression analysis to determine whether relationships were significant $\left({ }^{*} p<0.05\right)$, and we report the line of best fit to the data and the $\mathrm{R}^{2}$ value of the fit. Data showing actual lines of best fit can be found in Fig. 6. Note that work rate $\left(\mathrm{J} \mathrm{s}^{-1}\right)$ and power $(\mathrm{W})$ are synonymous 
understanding of the effect of passive-elastic ankle exoskeleton assistance on lower-limb joint mechanics and muscle activity and establish which factors drive changes in users' whole body metabolic rate. Traditionally, exoskeleton effects have been analyzed at a single speed where it is assumed that even with the addition of exoskeleton assistance, the stride time remains relatively consistent. The traditional approach calculates an average moment per stride (or period of interest) which is then compared to the metabolic rate (energy per second). For conditions with constant stride times, this approach may be sound. However, to enable the comparison of metabolic rate and other neuromechanical metrics across a wide range of exoskeleton stiffness and multiple speeds, the assumption of constant stride time is no longer sound, and the comparison of average moment per stride (no indication of time) to energy per time did not seem appropriate. Inspired by the field of integrative physiology and research findings from Taylor, Kram, and colleagues [36-42], we calculated the rate of the metric of interest (e.g. ankle moment, muscle activation) computed as the average of the metric per unit time (e.g. average moment per second, or average muscle activation per second). Upon inspection, the changes in rate metrics that were observed across exoskeleton stiffness and walking speed conditions were likely due to both changes in the average magnitude of the metrics and the duration of the stride (Supp. Tables 2-4). We used the rate of the metric of interest (average per second) for a more apt comparison to metabolic rate $\left(\mathrm{J} \mathrm{s}^{-1}\right)$ (See [43] for a similar approach).

With this approach, we sought to determine which factors drive changes in users' whole body metabolic rate across speeds. Whole body metabolic rate during walking can in part be attributed to the energetic cost of active muscle contraction (i.e., use of ATP for cycling cross-bridges) across the lower limbs. Recent work in muscle energetics shows that muscle stretchshorten cycles requires similar amounts of energy to an isometric contraction over the same duration which suggests that muscle energy use may be more closely tied to costs of force rather than work production [44]. In an exoskeleton hopping study, metabolic cost was reduced when muscle force was reduced but muscle work remained constant [45]. Thus, we anticipated that much of the metabolic improvement from an elastic ankle exoskeleton would be provided by reducing biological ankle joint moment and therefore plantarflexor muscle-tendon force and ultimately active muscle volume of the calf muscles [46]. This would suggest that applying more torque assistance to reducing biological muscle loading as much as possible should be the best way to maximize metabolic benefit of an exoskeleton.
Our results suggest that to some extent the simple solution that exoskeletons reduce biological joint moments, muscle-tendon forces and active muscle volume appears correct. Indeed, the conditions where we achieved a reduction in metabolic cost $\left(k_{\text {exo }}=50 \mathrm{Nm}\right.$ $\operatorname{rad}^{-1}$ at 1.25 and $1.75 \mathrm{~m} \mathrm{~s}^{-1}$ ) also exhibited measured reductions in biological ankle moment (Figs. $3 \mathrm{a}$ and $5 \mathrm{a}$, Supp. Figure 1B) and reduced soleus muscle activity (Figs. 3c and 5c, Supp. Figure 2A). However, our data also suggest that across walking speeds, more assistance (i.e., higher exoskeleton stiffness) is not better, and that when a 'sweet-spot' where metabolic benefit is achieved is found, it results from balancing appropriate amounts of exoskeleton torque assistance with deleterious side effects that can manifest via altered motor coordination, limb-joint kinetics and muscle-tendon dynamics [4750]. For example, when further evaluating users' physiological response to elastic ankle exoskeletons at each speed in isolation, qualitative trends in the effect of increasing device stiffness on 'non-local' neuromechanics (i.e., not ankle plantarflexors) were consistent and pointed toward potential metabolic penalties. Increasing exoskeleton assistance consistently resulted in increased MG, LG, and TA muscle activity (Supp. Figure 2B, C, D). In addition, at the knee, for each of the speeds we studied, we measured a substantial increase in muscle activation from the BFL (Supp. Figure 2E) that was accompanied by an expected concomitant shift from knee extension to knee flexion moment (Supp. Figure 3B). Despite these consistent qualitative, if not quantitative, trends in users' joint-level and muscle-level neuromechanical response to increasing exoskeleton stiffness, it was difficult to find a simple mechanical explanation for the differences in the observed relationships between exoskeleton stiffness and users' net metabolic rate at both slow and fast speeds ('bowl-shaped' at 1.25 and $1.75 \mathrm{~m} \mathrm{~s}^{-1}$ ) versus at the intermediate walking speed (monotonically increasing at $1.5 \mathrm{~m} \mathrm{~s}^{-1}$ ).

One possibility was that the ankle exoskeleton mechanical performance across stiffness could have been speed dependent, rendering it least effective at $1.5 \mathrm{~m} \mathrm{~s}^{-1}$. In fact, contrary to the dynamics of the biological ankle, where both the moment and power increase steadily with speed $[18,51]$, exoskeleton torque actually decreased with increasing walking speed (Supp. Figure 1B). These results are perhaps not surprising considering passive-elastic exoskeleton torque is driven by the user's ankle joint kinematics and peak ankle dorsiflexion is known to decreases with increasing walking speed [52]. Indeed, our results indicate that peak ankle dorsiflexion decreased by $11 \%$ in unassisted conditions across speed and by as much as $43 \%$ in the stiffest exoskeleton within a given speed. As a result, we observed very little increase in exoskeleton positive/negative power with speed 
and increases in exoskeleton power were small compared to increases in biological power calculated from our unassisted trials (47\%) and previous work performed over the same speed range (45\%) [18].

Despite the inability of our passive-elastic ankle device to increase its mechanical output with increasing speed, we found no relationship between exoskeleton positive power (= positive work rate) and users' net metabolic rate (Fig. 6, Table 1). This is in contrast to previous results from active devices, where exoskeleton mechanical power output seems to drive user benefit $[4,5,7,26$, 53]. Perhaps this incongruity stems from fundamental differences in the timing of assistance torque, as most active ankle systems are specifically designed to inject positive power to during late stance push-off. Our passive-elastic ankle exoskeletons apply torque to offload biological plantarflexor force with onset much earlier in stance. However, neither changes in users' biological positive power (i.e. work rate) or biological moment rate could explain the changes in net metabolic rate across all speeds (Fig. 6 and Table 1). The lack of relationship between changes in users' biological moment rate and metabolic rate may seem to contradict the idea that muscle force drives metabolic energy consumption; however, ankle moment is calculated as an externally measured net moment and does not account for cocontraction of antagonists muscles crossing the joint. Thus, an increase in co-contraction of dorsiflexors (e.g., TA) would result in underestimation of plantarflexor muscle force when derived from biological ankle moment. Indeed, we measured a significant increase in TA muscle activity with increasing exoskeleton stiffness which suggests an increase in co-contraction across the ankle. Therefore, in this study, changes in biological ankle moment were likely a poor representation of changes in plantarflexor muscle force and may help explain the poor relationship between changes in biological moment and changes in net metabolic rate.

A closer look at changes in ankle muscle activity reveals a nuanced trade-off between beneficial reductions in plantarflexor activity and costly increases in antagonist dorsiflexor activity across the range of exoskeleton stiffnesses we tested (Figs. 3c, 4c, and 5c). In fact, we find that the change in SOL+TA activation rate universally explains the bowl-shape net metabolic rate versus exoskeleton stiffness relationship at $1.25 \mathrm{~m} \mathrm{~s}^{-1}\left(\mathrm{R}^{2}=\right.$ $0.56)$ and $1.75 \mathrm{~m} \mathrm{~s}^{-1}\left(\mathrm{R}^{2}=0.69\right)$ and also the lack of bowl-shape relationship at $1.5 \mathrm{~m} \mathrm{~s}^{-1}\left(\mathrm{R}^{2}=0.64\right)$ (Fig. 6 and Table 1). Although there was no statistical linear effect of stiffness on SOL+TA activation, the intermediate walking speed $\left(1.50 \mathrm{~ms}^{-1}\right)$ was the only condition in which the data suggest that SOL+ TA activation was not reduced. While the data suggest that $\mathrm{SOL}$ activation rate decreased at $50 \mathrm{Nm} \mathrm{rad}^{-1}$ for all three speeds, TA activation rate was substantially increased at the intermediate speed $(18 \%)$ in comparison to the slight increase $(\sim 2 \%)$ in TA activation rate at slow $\left(1.25 \mathrm{~ms}^{-1}\right)$ and fast $\left(1.75 \mathrm{~ms}^{-1}\right)$ walking speeds. The changes in activation rate that were observed across exoskeleton stiffness and walking speeds were likely due to both changes in the average magnitude of the activation and the duration of the stride (Supp. Tables 2-4).

As mentioned earlier, and consistent with our previous experimental [10] and modeling/simulation [47] results for walking at $1.25 \mathrm{~m} \mathrm{~s}^{-1}$, our data here show that humans respond to increasing ankle exoskeleton stiffness with deleterious side effects manifesting via altered motor coordination (Supp. Figure 2), limb kinetics (Supp. Figs, 1, 3 , and 4), and muscle-tendon dynamics across the entire range of normal walking speeds $\left(1.25-1.75 \mathrm{~m} \mathrm{~s}^{-1}\right)$. But why is it that 'more is not better'? Or more explicitly, what physiological mechanisms may be preventing participants from converting larger and larger local reductions in biological plantarflexor muscle loading to metabolic savings? First, we note a trend toward diminishing returns. Although the exoskeleton stiffness increased by $67 \%$ between the $k_{\text {exo }}=150$ and $250 \mathrm{Nm} \mathrm{rad}^{-1}$ conditions the peak exoskeleton torque increased by only 13\% (Fig. 3a). Furthermore, we observe that participants compensated by decreasing the amount of ankle dorsiflexion (Supp. Figure 1), but the mechanism for this compensation is unclear. One potential explanation is that the user is unable to 'turn down' their muscle activation in proportion to the level of assistance provided. Joints (and limbs) tend to maintain constant stiffness during a given locomotion condition [54]. This is potentially accomplished through a combination of physiological sensors including length (spindle organs) and force (Golgi tendon organs) sensitive transducers that are responsible for maintaining a nearly constant ratio between changes in muscle force and length [54, 55]. In our data, ankle moment decreased from which we can predict a decrease in plantarflexor muscle force. On the other hand, studies suggest that muscle fascicle lengths become longer with increasing exoskeleton assistance [43, 47]. Thus, a conflict or mismatch in sensory feedback via Golgi tendon and muscle spindle organs may constrain motor adaptation in response to assistive forces. Much of walking is automatic and unconsciously controlled though spinal level central pattern generators, reflexes, cerebellar regulation, and the brainstem [56], and users of the exoskeleton may not be able to turn off their plantarflexors as would be required to obtain maximal benefit or coordination with the exoskeleton. Further work in humans and animals into the role of feedforward/feedback mechanisms and how they are modulated during perturbed walking would be insightful and help understand constraints on motor adaptation during walking with exoskeletons. 
Another potential side effect of applying exoskeleton assistance was that the normally efficient muscle-tendon dynamics could become 'detuned'. Muscles dynamics are governed by intrinsic muscle properties where force production and economy are dependent upon the length of a muscle and its contraction velocity [57, 58]. When exoskeleton assistance is applied, biological moment and thus muscle-tendon force decreases, while strain on the tendon decreases. Modeling and experimental studies of hopping $[45,48,59]$ and walking $[43,47,50]$ with ankle exoskeletons suggest that muscle lengths are in fact longer and undergo increased excursion with increased exoskeleton assistance, and this could limit muscle force capacity and metabolic economy. Additionally, the biological system may be resistant to increased muscle strain to avoid injury and compensations may arise to limit range of motion [60-62]. A closer look at the effect of elastic ankle assistance on plantarflexor muscle fascicle dynamics may give additional insights into how external forces applied in parallel with biological muscletendon units could be controlled to steer individual muscle dynamics $[43,46]$.

At the joint level, in early stance, the application of elastic exoskeleton torque did not significantly reduce the biological moment (Figs. 3aii, 4aii, and 5aii), but rather enhanced the amount of total ankle moment (i.e., augmentation) (Supp. Figure 1B). In late stance, on the other hand, the exoskeleton reduced the biological moment while the peak total ankle moment remained fairly constant (i.e., replacement) (Figs. 3aiii, 4aiii, and 5aiii). These findings raise the question if, as literature suggests, humans act to maintain a constant total ankle joint moment during walking [63] then why was the total ankle moment increased during the beginning of stance? One possible explanation is that the biarticular MG and LG muscles become 'overactive' to prevent hyperextension of the knee (Supp. Figure 2 B, C). Due to the kinetic chain within the lower limbs, the plantarflexion torque of the exoskeleton also generates an extension moment at the knee through dynamic coupling [64]. Perhaps as a compensation for the increasing knee extension moment with increasing exoskeleton stiffness, MG and LG increased activity that may have served as a countermeasure to push the knee towards flexion (Supp. Figure 2B, C; Supp. Figure 3B). The increased activation of the BFL, which is a knee flexor, also supports the idea that additional muscle activation was required for knee flexion (Supp. Figure 2E). The effect exists for the entire stride but may be more critical in early stance when the ground reaction force vector briefly passes in front of the knee. The gastrocnemius moment arm is also larger at the knee for more extended postures [65], giving it more leverage during early stance. It may be possible to avoid costly compensations by using biarticular exoskeleton designs that incorporate a knee component designed to act in a similar manner to the gastrocnemius and compensate for additional load on knee flexors derived from assistive ankle torques [66]. Alternatively, an exoskeleton with a non-linear or piecewise linear stiffness profile where ankle stiffness stayed low until the ground reaction force vector passed behind the knee could also be a potential solution. Unfortunately, delaying the onset angle for the exoskeleton spring or limiting stiffness also has the effect of limiting the amount of energy that can be stored and returned in the device.

Contrary to our expectation, we did not find that optimal exoskeleton stiffness increased substantially with walking speed. Based on the computed exoskeleton stiffness at minimum net metabolic rate from our fitted regressions, we estimate that the optimal stiffness for slow $\left(70 \mathrm{Nm} \mathrm{rad}^{-1}\right.$ at $\left.1.25 \mathrm{~m} \mathrm{~s}^{-1}\right)$ and fast $\left(79 \mathrm{Nm} \mathrm{rad}^{-1}\right.$ at $1.75 \mathrm{~m} \mathrm{~s}^{-1}$ ) walking speeds were similar. The increase in optimal exoskeleton stiffness that we measured (13\%) was not proportional to the estimated speed-dependent increase in ankle quasi-stiffness during plantarflexion (21\%) or dual-flexion (i.e., late dorsiflexion) (46\%) calculated from regression models derived from human walking data [24]. Instead, the $13 \%$ increase in optimal exoskeleton stiffness was in closer agreement with the $10 \%$ increase in ankle quasi-stiffness during the dorsiflexion phase [24]. This discrepancy can likely be explained by the role of muscle and tendon during stance. During late dorsi-flexion (dual-flexion) and plantarflexion, the contraction of the soleus muscle likely modulates the quasi-stiffness of the joint while the person adapts to walking speed. As walking speed increases, muscle activity increases, plantarflexion begins earlier, and quasi-stiffness in late stance is higher. In early stance during dorsiflexion, the soleus muscle produces force isometrically [20] and allows the Achilles tendon to stretch against it to store energy [22]. Thus, the Achilles tendon (and not concentric muscle contraction) is likely responsible for a large percentage of ankle joint rotational stiffness in early stance, and stiffness during this period is less likely to be actively modulated with speed. Similar to a biological tendon, our passive exoskeleton has no 'muscle' and is not capable of modulating stiffness in late stance to adapt to increased walking speeds. Therefore, in hindsight, it may not be that surprising that the optimal exoskeleton stiffness tracks speed-dependent changes in ankle quasi-stiffness during early stance dorsiflexion, when the biological tendon stiffness dominates the quasi-stiffness behavior of the ankle. A positive consequence of the relative invariance in optimal exoskeleton stiffness at slow and fast walking speeds is that even though low-powered clutch-spring 
systems are being developed that can switch stiffness step by step [67], designing for variable speed conditions becomes less challenging and may not even be necessary.

The changes in SOL+TA activation explain why metabolic reduction was not obtained at intermediate walking speed $\left(1.5 \mathrm{~m} \mathrm{~s}^{-1}\right)$; however, the mechanism behind the difference in muscle response at intermediate speed versus the other speeds is less clear. Changes in joint mechanics were similar for all three speeds with differences observed in terms of timing or amplitude that were merely exacerbated at the highest walking speed. This suggests to us that perhaps the differential effect of exoskeleton stiffness on users' net metabolic rate at $1.5 \mathrm{~m}$ $\mathrm{s}^{-1}$ was due to muscle-tendon dynamics that were not evident in joint-level data. Previous literature suggests that preferred walking speed in adults is close to $1.42 \mathrm{~m}$ $\mathrm{s}^{-1}$, and this speed aligns closely with that which minimizes the metabolic cost of transport [28]. Humans also select step frequencies that reduce energetic cost of walking $[68,69]$. The structure of the ankle is important for maximizing muscle efficiency [70, 71] (i.e., minimizing metabolic cost) and models of walking have suggested that walking efficiency is maximized when step length and frequency are matched to target ankle stiffness [72]. Therefore, it is possible that, nominally, ankle plantarflexors are preferentially 'tuned' to participants' preferred walking speed. In this study, preferred walking speed collected from participants using a $10 \mathrm{~m}$ over ground test was $1.39 \pm 0.04 \mathrm{~m} \mathrm{~s}^{-1}$ and the average metabolic cost of transport with no exoskeleton assistance at $1.25,1.50$, and $1.75 \mathrm{~m} \mathrm{~s}^{-1}$ was $2.5 \pm 0.11,2.67 \pm 0.13$, and $3.22 \pm 0.15 \mathrm{~J} \mathrm{~m}^{-1}$ respectively. Thus, we suspect that the muscle-level 'detuning' associated with the exoskeleton assistance may have been more pronounced at the $1.5 \mathrm{~m}$ $\mathrm{s}^{-1}$ walking speed due to its proximity to the preferred (and perhaps most economical) speed.

One difference between these study results and our previous study on passive elastic ankle exoskeletons was that the optimal stiffness reported in this study $(50 \mathrm{Nm}$ $\left.\operatorname{rad}^{-1}\right)$ is substantially lower than the reported optimal stiffness from the prior work (180 $\left.\mathrm{Nm} \mathrm{rad}^{-1}\right)$ [10]. However, the stiffness values previously reported reflected the stiffness of exoskeleton spring elements rather than the stiffness of the whole exoskeleton system. Conversely, the exoskeleton testbed we used here (Fig. 1) imposes a desired torque/angle relationship rather than relying on physical components to provide stiffness making it less sensitive to structural stiffness and deformation of the exoskeleton frame. When deformation in the previous system is accounted for by measuring stiffness from the exoskeleton's torque/angle relationship, the optimal stiffness for $1.25 \mathrm{~m} \mathrm{~s}^{-1}$ walking speed was reduced to $\sim 80 \mathrm{Nm} \mathrm{rad}$. This is close to the optimal value we found here based on the stiffness at minimum net metabolic power from the regression at the same speed $\left(69 \mathrm{Nm} \mathrm{rad}^{-1}\right)$. We note that there may still have been some movement between the human and device that was not accounted for in our system, but we believe that stiffness values reported here give a more accurate portrayal of the applied rotational stiffness than had been previously reported.

We acknowledge that our study has limitations. In terms of the hardware, our exoskeleton emulator behaved more like an ideal spring rather than a physical spring or a biological spring, which would be expected to dissipate energy in each cycle. Achilles tendon hysteresis, for example, could be as much as $15 \%$ per cycle [73], though the true value may be smaller and is still debated [74]. Thus, although our 'exo-tendon' did not emulate the exact energy cycle of a biological tendon, our intention was to tightly control stiffness, and vary it systematically, so that we could measure its effect on users' physiological response. Small amounts of work were generated/dissipated, but we estimate the impact of these amounts of mechanical energy were less than $0.1 \%$ of the total net metabolic power. Future work could consider damping in addition to stiffness in the exoskeleton impedance control law to regulate the amount of energy dissipated by the exoskeleton.

Another limitation, in terms of protocol, was that rather than build a streamlined, low-mass and portable version of our device (e.g., as was done in [10]) we opted to use a high-powered, tethered ankle exoskeleton system to emulate and assess the effect of passiveelastic exoskeleton stiffness on the neuromechanics and energetics of walking across speeds. Our primary goal here was to employ a framework to rapidly and robustly test the specific effects of ankle exoskeleton stiffness independent of the mass and inertia of the device with high repeatability. This choice restricted our ability to definitively claim that the savings we measured with respect to the no assistance baseline condition (i.e., $\mathrm{k}_{\mathrm{exo}}=$ $0 \mathrm{Nm} \mathrm{rad}{ }^{-1}$ or zero-torque) would transfer to real savings with respect to normal walking in a portable version of the device using similar stiffness. For the tethered exoskeleton emulator we used here (Fig. 1), the increase in metabolic cost due to merely donning the system was on average $19 \%$ (Supp. Table 1). We note, however, that our previous work indicates it is possible to build a passive-elastic system with negligible added mass cost [10], making the effect of the spring stiffness the primary variable of interest, and perhaps a more useful benchmark for extrapolating our results to expected performance on a portable analogue. Thus, our results suggest it should be possible to reduce metabolic cost of walking at both slow and fast speeds by $\sim 5 \%$ using an unpowered, passive- elastic ankle 
exoskeleton with fixed rotational stiffness $\sim 70-80 \mathrm{Nm}$ $\operatorname{rad}^{-1}$ (Fig. 2).

\section{Conclusions}

In a move toward 'real-world' application, we examined whether the metabolic benefit of walking with unpowered elastic ankle exoskeletons extends across a range of walking speeds, and if so, whether the optimal exoskeleton stiffness was speed dependent. We showed that users were able to achieve a reduction in net metabolic power at slow ( $4.2 \%$ at $\left.1.25 \mathrm{~m} \mathrm{~s}^{-1}\right)$ and fast $(4.7 \%$ at 1.75 $\mathrm{m} \mathrm{s}^{-1}$ ) walking speeds but were not able to get a metabolic reduction at $1.5 \mathrm{~m} \mathrm{~s}^{-1}$. These data suggest that the effect of elastic exoskeleton assistance varies with walking speed and is potentially limited at speeds close to the preferred (i.e., nominally metabolically optimal) walking speed. In addition, contrary to our initial hypothesis, we found no appreciable increase in optimal exoskeleton stiffness with increasing walking speed. For both slow and fast walking speeds, optimal stiffness was between 50 and $100 \mathrm{Nm} \mathrm{rad}^{-1}$. These findings help provide an initial 'road-map' for how ankle exoskeleton impedance should be adjusted (or not!) to match the changing mechanical demands of locomotion in 'real-world' environments. We suspect that studies designed to look 'under the skin' using computational modeling or ultrasound imaging and examine how exoskeleton stiffness influences biological muscle-tendon dynamics will yield further insight about how to optimally blend the impedance of an exoskeleton with that of a human joint.

\section{Supplementary information}

Supplementary information accompanies this paper at https://doi.org/10. 1186/s12984-020-00703-4.

Additional file 1: Supplementary Figures and Tables.
Supplementary Figure 1: Ankle joint mechanics across stiffness and
speed. Supplementary Figure 2: Muscle activity across stiffness and
speed. Supplementary Figure 3: Knee joint mechanics across stiffness
and speed. Supplementary Figure 4: Hip joint mechanics across
stiffness and speed. Supplementary Figure 5: Net metabolic rate
during exoskeleton training at $1.25 \mathrm{~m} \mathrm{~s}^{-1}$. Supplementary Table 1:
Steady-state net metabolic rate for each subject, condition, and speed.
Supplementary Table 2: Summary statistics for exoskeleton stiffness ef-
fect at $1.25 \mathrm{~m} \mathrm{~s}^{-1}$. Supplementary Table $\mathbf{3}$ : Summary statistics for exo-
skeleton stiffness effect at $1.50 \mathrm{~m} \mathrm{~s}^{-1}$. Supplementary Table 4: Summary
statistics for exoskeleton stiffness effect at $1.75 \mathrm{~m} \mathrm{~s}^{-1}$.

\section{Abbreviations}

EMG: Electromyography; GRF: Ground reaction force; SOL: Soleus; MG: Medial Gastrocnemius; LG: Lateral Gastrocnemius; TA: Tibialis Anterior; BFL: Biceps Femoris Longhead; RF: Rectus Femoris; IRB: Institutional review board

\section{Acknowledgements}

We thank S. Steele-Pardue and T. Giest for assistance in assembling the device, T. Giest, J. McCall, and S. Philius for data collection, T. Dick for data collection and manuscript editing, F. Shaw for data analysis.

\section{Authors' contributions}

RWN and GSS contributed equally to study design and direction; RWN designed and fabricated the device; RWN conducted human locomotion experiments; RWN and GSS analyzed data; RWN and GSS drafted the manuscript; RWN and GSS edited the manuscript. All the authors read and approved the final version of the manuscript

\section{Funding}

Funding for this research was provided by grant R01NR014756 from the National Institute of Nursing Research of the National Institutes of Health to G.S.S. The content is solely the responsibility of the authors and does not necessarily represent the official views of the National Institutes of Health or other funding agencies listed.

\section{Availability of data and materials}

Source data from this study in .mat and .txt format and an associated readme.txt for navigating it are available for download at: http://pwp.gatech. edu/hpl/archival-data-from-publications/

Ethics approval and consent to participate

Data collection procedures were approved by the University of North Carolina at Chapel Hill IRB, and all participants signed an IRB approved consent form before data collections.

\section{Consent for publication}

Not Applicable.

\section{Competing interests}

The authors have no competing interest related to this work.

\section{Author details}

${ }^{1}$ Joint Department of Biomedical Engineering, UNC Chapel Hill and NC State University, Raleigh, NC, USA. ${ }^{2}$ John A. Paulson School of Engineering and Applied Sciences, Harvard University, Cambridge, MA 02138, USA. ${ }^{3}$ Wyss Institute for Biologically Inspired Engineering, Harvard University, Cambridge, MA 02138, USA. ${ }^{4}$ George W. Woodruff School of Mechanical Engineering and School of Biological Sciences, Georgia Institute of Technology, Atlanta, GA 30332, USA

Received: 5 January 2020 Accepted: 21 May 2020

Published online: 15 June 2020

\section{References}

1. Malcolm P, Derave W, Galle S, De Clercq D. A simple exoskeleton that assists plantarflexion can reduce the metabolic cost of human walking. PLoS One. 2013;8(2):e56137.

2. Sawicki GS, Beck ON, Kang I, Young AJ. The exoskeleton expansion: improving walking and running economy. J NeuroEng Rehabil. 2020;17(1): 25.

3. Mooney LM, Rouse EJ, Herr HM. Autonomous exoskeleton reduces metabolic cost of walking. Conf Proc IEEE Eng Med Biol Soc. 2014;2014: 3065-8

4. Mooney LM, Rouse EJ, Herr HM. Autonomous exoskeleton reduces metabolic cost of human walking during load carriage. J Neuroeng Rehabil. 2014;11:80.

5. Mooney LM, Herr HM. Biomechanical walking mechanisms underlying the metabolic reduction caused by an autonomous exoskeleton. J Neuroeng Rehabil. 2016:13:4.

6. Galle S, Malcolm P, Collins SH, De Clercq D. Reducing the metabolic cost of walking with an ankle exoskeleton: interaction between actuation timing and power. J Neuroeng Rehabil. 2017;14(1):35

7. Zhang J, Fiers P, Witte KA, Jackson RW, Poggensee KL, Atkeson CG, et al. Human-in-the-loop optimization of exoskeleton assistance during walking. Science. 2017;356(6344):1280-4.

8. Ding Y, Kim M, Kuindersma S, Walsh CJ. Human-in-the-loop optimization of hip assistance with a soft exosuit during walking. Sci Robotics. 2018;3(15): eaar5438.

9. Lim B, Lee J, Jang J, Kim K, Park YJ, Seo K, et al. Delayed output feedback control for gait assistance with a robotic hip exoskeleton. IEEE Trans Robot. 2019;35(4):1055-62. 
10. Collins SH, Wiggin MB, Sawicki GS. Reducing the energy cost of human walking using an unpowered exoskeleton. Nature. 2015;522(7555):212-5.

11. Galle S, Malcolm P, Derave W, De Clerca D. Enhancing performance during inclined loaded walking with a powered ankle-foot exoskeleton. Eur J Appl Physiol. 2014;114(11):2341-51.

12. Sawicki GS, Ferris DP. Mechanics and energetics of incline walking with robotic ankle exoskeletons. J Exp Biol. 2009;212(Pt 1):32-41.

13. Quinlivan B, Lee S, Malcolm P, Rossi D, Grimmer M, Siviy C, et al. Assistance magnitude versus metabolic cost reductions for a tethered multiarticular soft exosuit. Science Robotics. 2017;2(2):eaah4416.

14. Verstraten T, Beckerle P, Furnémont R, Mathijssen G, Vanderborght B, Lefeber D. Series and parallel elastic actuation: impact of natural dynamics on power and energy consumption. Mech Mach Theory. 2016;102:232-46.

15. Grimmer M, Eslamy M, Seyfarth A. Energetic and peak power advantages of series elastic actuators in an actuated prosthetic leg for walking and running. Actuators. 2014;3(1):1-19.

16. Grimmer M, Seyfarth A. Stiffness adjustment of a series elastic actuator in an ankle-foot prosthesis for walking and running: the trade-off between energy and peak power optimization. IEEE Int Conf Robot Automation. 2011;2011: 1439-44.

17. Wang S, Van Dijk W, van der Kooij H. Spring uses in exoskeleton actuation design. IEEE Int Conf Rehab Robot. 2011;2011:1-6.

18. Farris DJ, Sawicki GS. The mechanics and energetics of human walking and running: a joint level perspective. J R Soc Interface. 2012;9(66):110-8.

19. Kuo AD, Donelan JM, Ruina A. Energetic consequences of walking like an inverted pendulum: step-to-step transitions. Exerc Sport Sci Rev. 2005;33(2): 88-97.

20. Ishikawa M, Komi PV, Grey MJ, Lepola V, Bruggemann GP. Muscle-tendon interaction and elastic energy usage in human walking. J Appl Physiol (1985). 2005;99(2):603-8.

21. Lai A, Lichtwark GA, Schache AG, Lin YC, Brown NA, Pandy MG. In vivo behavior of the human soleus muscle with increasing walking and running speeds. J Appl Physiol (1985). 2015;118(10):1266-75.

22. Farris DJ, Sawicki GS. Human medial gastrocnemius force-velocity behavio shifts with locomotion speed and gait. Proc Natl Acad Sci U S A. 2012; 109(3):977-82.

23. Bregman DJ, van der Krogt MM, de Groot V, Harlaar J, Wisse M, Collins SH. The effect of ankle foot orthosis stiffness on the energy cost of walking: a simulation study. Clin Biomech (Bristol, Avon). 2011;26(9):955-61.

24. Shamaei K, Sawicki GS, Dollar AM. Estimation of quasi-stiffness and propulsive work of the human ankle in the stance phase of walking. PLoS One. 2013;8(3):e59935.

25. Caputo JM, Collins SH. A universal ankle-foot prosthesis emulator for human locomotion experiments. J Biomech Eng. 2014;136(3):035002.

26. Jackson RW, Collins SH. An experimental comparison of the relative benefits of work and torque assistance in ankle exoskeletons. J Appl Physiol (1985). 2015:119(5):541-57.

27. Zhang J, Cheah CC, Collins SH. Experimental comparison of torque control methods on an ankle exoskeleton during human walking. In 2015 IEEE International Conference on Robotics and Automation (ICRA) 2015 May 26 (pp. 5584-5589). IEEE.

28. Browning RC, Baker EA, Herron JA, Kram R. Effects of obesity and sex on the energetic cost and preferred speed of walking. J Appl Physiol (1985). 2006; 100(2):390-8.

29. Neptune RR, Sasaki K. Ankle plantar flexor force production is an important determinant of the preferred walk-to-run transition speed. J Exp Biol. 2005; 208(5):799-808.

30. Schmidt RA, Lee T. Motor control and learning: human kinetics; 1988.

31. Sawicki GS, Ferris DP. Mechanics and energetics of level walking with powered ankle exoskeletons. J Exp Biol. 2008;211(Pt 9):1402-13.

32. Gordon KE, Ferris DP. Learning to walk with a robotic ankle exoskeleton. J Biomech. 2007:40(12):2636-44.

33. Galle S, Malcolm P, Derave W, De Clercq D. Adaptation to walking with an exoskeleton that assists ankle extension. Gait Posture. 2013:38(3):495-9.

34. Zelik KE, Takahashi KZ, Sawicki GS. Six degree-of-freedom analysis of hip, knee, ankle and foot provides updated understanding of biomechanical work during human walking. J Exp Biol. 2015;218(Pt 6):876-86.

35. Brockway JM. Derivation of formulae used to calculate energy expenditure in man. Human Nutr Clin Nutr. 1987;41(6):463-71.

36. Roberts TJ, Chen MS, Taylor CR. Energetics of bipedal running. II. Limb design and running mechanics. J Exp Biol. 1998;201(Pt 19):2753-62.
37. Roberts TJ, Kram R, Weyand PG, Taylor CR. Energetics of bipedal running. I. Metabolic cost of generating force. J Exp Biol. 1998;201(Pt 19):2745-51.

38. Taylor CR. Relating mechanics and energetics during exercise. Adv Vet Sci Comp Med. 1994;38A:181-215.

39. Griffin TM, Roberts TJ, Kram R. Metabolic cost of generating muscular force in human walking: insights from load-carrying and speed experiments. J Appl Physiol (1985). 2003;95(1):172-83.

40. Biewener AA, Farley CT, Roberts TJ, Temaner M. Muscle mechanical advantage of human walking and running: implications for energy cost. J Appl Physiol. 2004;97(6):2266-74.

41. Kipp S, Grabowski AM, Kram R. What determines the metabolic cost of human running across a wide range of velocities? J Exp Biol. 2018;221(18).

42. Kram R, Taylor CR. Energetics of running: a new perspective. Nature. 1990; 346(6281):265-7.

43. Nuckols RW, Dick TJM, Beck ON, Sawicki GS. Ultrasound imaging links soleus muscle neuromechanics and energetics during human walking with elastic ankle exoskeletons. Sci Rep. 2020;10(1):3604.

44. Holt NC, Roberts TJ, Askew GN. The energetic benefits of tendon springs in running: is the reduction of muscle work important? J Exp Biol. 2014;217(Pt 24):4365-71.

45. Farris DJ, Robertson BD, Sawicki GS. Elastic ankle exoskeletons reduce soleus muscle force but not work in human hopping. J Appl Physiol (Bethesda, Md: 1985). 2013;115(5):579-85.

46. Beck ON, Punith LK, Nuckols RW, Sawicki GS. Exoskeletons improve locomotion economy by reducing active muscle volume. Exerc Sport Sci Rev. 2019;47(4):237-45.

47. Sawicki GS, Khan NS. A simple model to estimate Plantarflexor muscletendon mechanics and energetics during walking with elastic ankle exoskeletons. IEEE Trans Biomed Eng. 2016;63(5):914-23.

48. Robertson BD, Farris DJ, Sawicki GS. More is not always better: modeling the effects of elastic exoskeleton compliance on underlying ankle muscletendon dynamics. Bioinspir Biomim. 2014;9(4):046018.

49. Steele KM, Jackson RW, Shuman BR, Collins SH. Muscle recruitment and coordination with an ankle exoskeleton. J Biomech. 2017:59:50-8.

50. Jackson RW, Dembia CL, Delp SL, Collins SH. Muscle-tendon mechanics explain unexpected effects of exoskeleton assistance on metabolic rate during walking. J Exp Biol. 2017;220(Pt 11):2082-95.

51. Winter DA. Kinematic and kinetic patterns in human gait: variability and compensating effects. Hum Mov Sci. 1984;3(1):51-76.

52. Lelas JL, Merriman GJ, Riley PO, Kerrigan DC. Predicting peak kinematic and kinetic parameters from gait speed. Gait Posture. 2003;17(2):106-12.

53. Grimmer M, Quinlivan BT, Lee S, Malcolm P, Rossi DM, Siviy C, et al. Comparison of the human-exosuit interaction using ankle moment and ankle positive power inspired walking assistance. J Biomech. 2019;83:76-84.

54. McMahon TA. Muscles, reflexes, and locomotion. Princeton: Princeton; 1984

55. Roberts TJ. The integrated function of muscles and tendons during locomotion. Comp Biochem Physiol A Mol Integr Physiol. 2002;133(4):1087-99.

56. Enoka RM. Neuromechanics of human movement: human kinetics; 2008.

57. Hill AV. Length of muscle, and the heat and tension developed in an isometric contraction. J Physiol. 1925;60(4):237-63.

58. Hill AV. The heat of shortening and the dynamic constants of muscle. Proc R Soc Lond B Biol Sci. 1938;126(843):136-95.

59. Farris DJ, Hicks JL, Delp SL, Sawicki GS. Musculoskeletal modelling deconstructs the paradoxical effects of elastic ankle exoskeletons on plantar-flexor mechanics and energetics during hopping. J Exp Biol. 2014; 217(Pt 22):4018-28.

60. Morgan DL. New insights into the behavior of muscle during active lengthening. Biophys J. 1990;57(2):209-21.

61. Morgan DL, Allen DG. Early events in stretch-induced muscle damage. J Appl Physiol (1985). 1999;87(6):2007-15.

62. Allinger $\mathrm{TL}$, Epstein $\mathrm{M}$, Herzog W. Stability of muscle fibers on the descending limb of the force-length relation. A theoretical consideration. J Biomech. 1996;29(5):627-33.

63. Kao $P C$, Lewis $C L$, Ferris $D P$. Invariant ankle moment patterns when walking with and without a robotic ankle exoskeleton. J Biomech. 2010;43(2):203-9.

64. Zajac FE, Neptune RR, Kautz SA. Biomechanics and muscle coordination of human walking. Part I: introduction to concepts, power transfer, dynamics and simulations. Gait Posture. 2002;16(3):215-32.

65. Visser J, Hoogkamer J, Bobbert M, Huijing P. Length and moment arm of human leg muscles as a function of knee and hip-joint angles. Eur J Appl Physiol Occup Physiol. 1990;61(5):453-60. 
66. Malcolm P, Galle S, Derave W, De Clercq D. Bi-articular knee-ankle-foot exoskeleton produces higher metabolic cost reduction than weightmatched mono-articular exoskeleton. Front Neurosci. 2018;12:69.

67. Diller S, Majidi C, Collins SH, editors. A lightweight, low-power electroadhesive clutch and spring for exoskeleton actuation. 2016 IEEE International Conference on Robotics and Automation (ICRA); 2016 16-21 May 2016.

68. Selinger JC, O'Connor SM, Wong JD, Donelan JM. Humans can continuously optimize energetic cost during walking. Curr Biol. 2015;25(18):2452-6.

69. Minetti AE, Capelli C, Zamparo P, di Prampero PE, Saibene F. Effects of stride frequency on mechanical power and energy expenditure of walking. Med Sci Sports Exerc. 1995;27(8):1194-202.

70. Lichtwark GA, Wilson AM. Optimal muscle fascicle length and tendon stiffness for maximising gastrocnemius efficiency during human walking and running. J Theor Biol. 2008;252(4):662-73.

71. Lichtwark GA, Wilson AM. Is Achilles tendon compliance optimised for maximum muscle efficiency during locomotion? J Biomech. 2007;40(8): 1768-75.

72. Zelik KE, Huang TW, Adamczyk PG, Kuo AD. The role of series ankle elasticity in bipedal walking. J Theor Biol. 2014;346:75-85.

73. Shadwick RE. Elastic energy storage in tendons: mechanical differences related to function and age. J Appl Physiol (1985). 1990;68(3):1033-40.

74. Lichtwark GA, Cresswell AG, Ker RF, Reeves ND, Maganaris CN, Magnusson $\mathrm{SP}$, et al. Commentaries on viewpoint: on the hysteresis in the human Achilles tendon. J Appl Physiol. 2013;114(4):518-20.

\section{Publisher's Note}

Springer Nature remains neutral with regard to jurisdictional claims in published maps and institutional affiliations.

Ready to submit your research? Choose BMC and benefit from:

- fast, convenient online submission

- thorough peer review by experienced researchers in your field

- rapid publication on acceptance

- support for research data, including large and complex data types

- gold Open Access which fosters wider collaboration and increased citations

- maximum visibility for your research: over $100 \mathrm{M}$ website views per year

At BMC, research is always in progress.

Learn more biomedcentral.com/submissions 